\title{
Aeroelastic Uncertainty Quantification of a Low-Boom Aircraft Configuration
}

\author{
Ben D. Phillips* and Thomas K. West IV ${ }^{\dagger}$ \\ NASA Langley Research Center, Hampton, VA, 23681
}

\begin{abstract}
As the state of the art in uncertainty quantification for low-boom aircraft advances, the underlying assumption of a rigid airframe must be revisited. The goal of this research is to investigate the impact of uncertainties in aeroelastic deformation of a low-boom aircraft on ground noise. Variations in structural properties and uncertainties in loading, derived from flight conditions, both factor into the overall aeroelastic deformation and subsequently the ground noise. Incorporation of these aeroelastic uncertainties in the prediction of ground noise during the design phase can lead to improved robustness. In this paper, a review of methodologies and techniques employed in low-boom uncertainty quantification will be given. In addition, methods for aeroelastic uncertainty quantification are integrated into the previous work and a generalized set of procedures is established. In a case study implementing the analysis procedures, ground noise generated from a static aeroelastic deformed low-boom aircraft increased slightly over that from the undeformed geometry for both undertrack and offtrack angles. Ground noise sensitivities to uncertainties in near field conditions and structural parameters varied significantly with atmospheric profiles. Shifts in confidence interval width in addition to shifts in deterministic values of ground noise were observed while varying atmospheric conditions.
\end{abstract}

\section{Nomenclature}

$\begin{array}{llll}\text { ASEL } & \text { A-Weighted Sound Exposure Level }(\mathrm{dB}) & D & \text { Total Variance } \\ \text { CDF } & \text { Cumulative Distribution Function } & D_{T} & \text { Corrected Total Variance } \\ \text { CFD } & \text { Computational Fluid Dynamics } & D_{i} & \text { Variance of Principle Component } i \\ \text { CSEL } & \text { C-Weighted Sound Exposure Level }(\mathrm{dB}) & \Delta & \text { Difference } \\ \text { CST } & \text { Commercial Supersonic Technology } & \Delta P & \text { Differential Pressure } \\ \text { FAA } & \text { Federal Aviation Administration } & \delta_{i} & \text { Nodal Displacement } \\ \text { FAST } & \text { Fourier Amplitude Sensitivity Testing } & E & \text { Modulus of Elasticity } \\ \text { FEM } & \text { Finite Element Model } & F & \text { PCE Response } \\ \text { FSI } & \text { Fluid-Structure Interaction } & f_{o} & \text { Mean } \\ \text { HDMR } & \text { High Dimensional Model Representation } & G & \text { Shear Modulus } \\ \text { OML } & \text { Outer Mold Line } & M & \text { Mach Number } \\ \text { PCE } & \text { Polynomial Chaos Expansion } & P & \text { Pressure } \\ \text { PDF } & \text { Probability Density Function } & \Psi_{i} & \text { PCE Basis Functions } i^{t h} \text { Mode } \\ \text { PLdB } & \text { Perceived Loudness } & R & \text { Distance Along Near Field Sensor } \\ \text { UQ } & \text { Uncertainty Quantification } & R F & \text { Reflection Factor } \\ A_{i} & \text { PCE Deterministic Component } i^{t h} \text { Mode } & R H & \text { Relative Humidity } \\ \alpha & \text { Angle of Attack } & S_{i} & \text { Structural Parameter } i \\ a & \text { Significance Level } & \xi & \text { PCE Random Variables } \\ d & \text { PCE Deterministic Variables } & t & \text { Time }\end{array}$

*Aerospace Engineer, Aeronautics Systems Analysis Branch, Systems Analysis and Concepts Directorate, Member AIAA.

${ }^{\dagger}$ Aerospace Engineer, Vehicle Analysis Branch, Systems Analysis and Concepts Directorate, Member AIAA. 


\section{Introduction}

The concept of a commercial supersonic transport is not a new one as NASA research on the topic dates back to 1959. However, even with the many decades of research dedicated to advancing the state of the art, only two commercial supersonic transports have ever carried passengers. This is due in part to the prohibition on overland supersonic flight enacted in 1973, which remains in place today. Over the past decade, there have been heavy investments in the development of computational tools for sonic boom prediction and low-boom vehicle design. A current technical challenge for the NASA Commercial Supersonic Technology (CST) project is the development of design tools that enable the integrated design of a low-boom aircraft. A crucial portion of this tool development is the consideration of uncertainties in the prediction of the perceived loudness on the ground. ${ }^{1}$

The computation of aircraft sonic boom transmission to the ground starts with the estimation, through experimentation or simulation, of a pressure wave form in the near field, approximately three to five body lengths below the aircraft to ensure that three dimensional effects around the body are resolved. The near field pressure waveform is then propagated through the atmosphere by using an augmented Burgers' equation. $^{2}$ This procedure has two distinct areas where uncertainties are manifested: first, in the near field pressure waveform, where uncertainty is driven by uncertainties in the simulation or experiment; and second, in the ground signal, where uncertainty is driven by propagation uncertainties (which are confounded by the near field uncertainties). Figure 1 depicts the propagation of a pressure waveform to the ground.

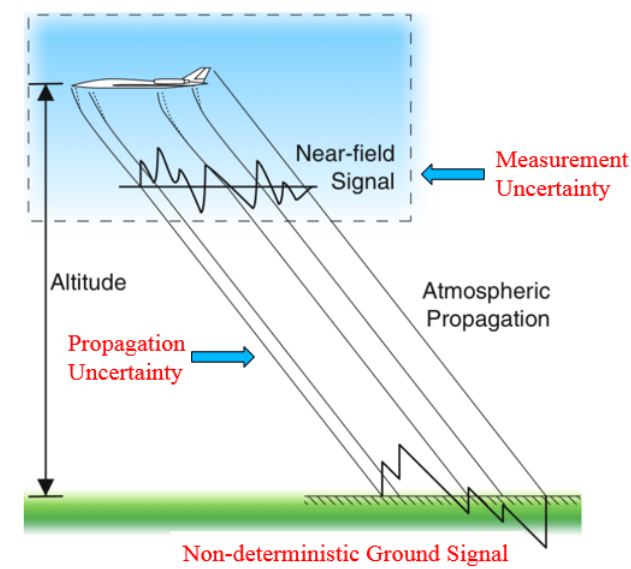

Figure 1: Propagation of the pressure waveform to the ground. ${ }^{3,4}$

Previous research has been dedicated to the investigation of uncertainty in the prediction of low-boom aircraft signatures for implementation in certification procedures. West et al..$^{5,6}$ have demonstrated efficient methods for certification prediction of low-boom aircraft. That work utilized a non-intrusive polynomial chaos approach in conjunction with novel dispersion methods outlined by Pinier ${ }^{7}$ to place statistically defensible uncertainty bounds on both the near field pressure waveform and overall perceived noise levels on the ground. The methodology employed by West is applicable to both experimentally and numerically generated signatures. In addition, his work assisted in validation of CFD generated near field pressure waveforms and attempted to define a framework for certification prediction under uncertainty. Previous research conducted by Phillips and West ${ }^{8}$ has shown that small changes in the outer mold line (OML) due to uncertainties in control surface deflections can have a significant impact on the overall uncertainty in ground noise. The impact of such small changes in OML indicates that aeroelastic structural deformation could also have a significant impact on overall ground noise. Nikbay et al. ${ }^{9}$ previously investigated the impact of aeroelastic uncertainties on overall ground noise. Initial results from Nikbay's work also illustrate that uncertainties in structural parameters (material properties) are significant in the overall uncertainty of noise on the ground. Nikbay's results indicate the importance of uncertainties in structural loading due to uncertainties in flight conditions, as well as establishing an iterative procedure to determine a converged loading condition.

The objective of this research is to incorporate a robust assessment of aeroelastic and structural uncertainties into the existing framework for low-boom aircraft configurations. An iterative fluid-structure interaction to determine accurate static aeroelastic deformation driven by uncertain flight conditions will be 
investigated. Recent work by Phillips and West $^{8}$ demonstrating the decoupling of uncertainties of the near field and atmospheric propagation will be employed to better model the uncertainties in the ground signature. This work will help establish the framework for a set of procedures in which all phases of supersonic commercial flight can be analyzed under uncertainty for certification prediction. ${ }^{5}$

The following section gives a brief overview of uncertainty quantification as it relates to low-boom prediction. Methods implemented in this research will also be detailed. Section III details the computational setup of the aeroelastic analysis followed by section IV which outlines the uncertainty modeling and propagation approaches. Results and important conclusions are discussed in sections V and VI, respectively.

\section{Methodology}

Generally speaking, uncertainty quantification (UQ) encompasses the study of uncertainties in input parameters on the output or response of a process or simulation. UQ can vary in scope by including only a single model, or multiple models of varying fidelity and experimental data. The overarching objective of UQ is to create a more robust design process by identifying and reducing uncertainties. An important facet of UQ is the proper characterization and treatment of the simulation input uncertainties. The input uncertainties in the analysis of sonic boom signatures can be divided into two categories: aleatory and epistemic. Aleatory uncertainty is inherent variation associated with a parameter, physical system, or environment and is referred to as irreducible or stochastic uncertainty. Epistemic uncertainty is a result of imperfect knowledge or ignorance of the system and is referred to as subjective uncertainty. ${ }^{4}{ }^{10}$ Examples of epistemic uncertainties in the analysis of boom signatures include the ground elevation and reflection factor whereas aleatory uncertainties include atmospheric conditions such as humidity, temperature, and wind. To perform a UQ study for sonic boom generation and propagation, multiple methods are necessary. The overall architecture for this study followed the second-order probability approach. To characterize the near field uncertainty, non-intrusive polynomial chaos with point-collocation was utilized. Then, through Fourier dispersions, the near field uncertainty was passed to the atmospheric propagation. Finally, to characterize the overall uncertainty, Monte Carlo variance decomposition was performed. The following three sections will detail the specific UQ methods adapted for this work.

\section{A. Second-Order Probability}

To propagate uncertainty through the model, the second-order probability approach outlined by Eldred and Swiler ${ }^{11}$ for the treatment of mixed aleatory and epistemic uncertainties was employed. A flowchart of the method is shown in Figure 2. In this application of the second-order probability approach, the black-box simulation is a computational fluid dynamics code with a fluid-structure interaction coupled finite element analysis and an atmospheric propagation code.

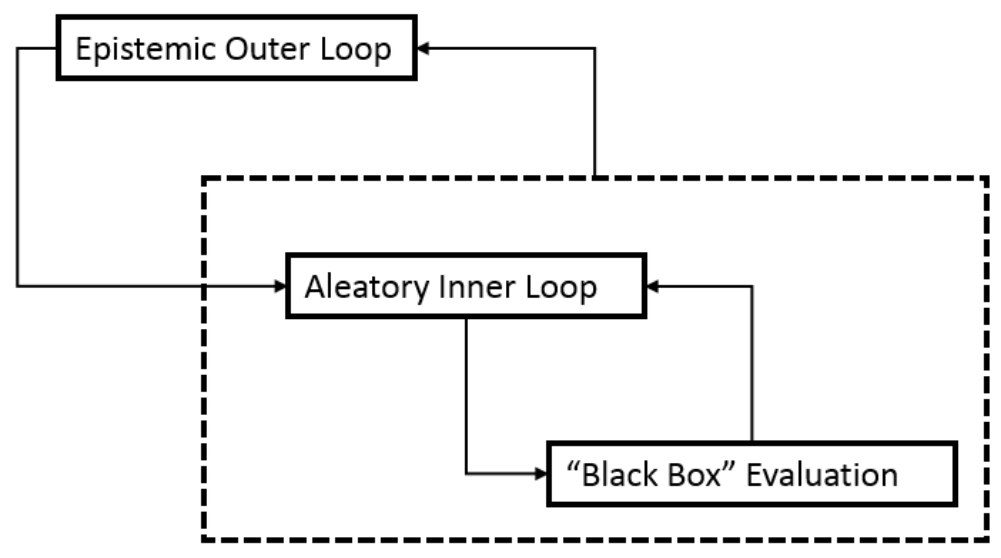

Figure 2: Second-Order probability architecture.

For each set of epistemic uncertainties, a cumulative distribution function can be generated from the set of associated aleatory uncertainties as seen in Figure 3. The probability box (P-Box) plot shows the family 
of cumulative distribution functions (CDF) generated from the second-order probability approach. To determine a $95 \%$ confidence interval on the perceived ground noise, the lowest perceived noise level extracted from a $\mathrm{CDF}$ at the $2.5 \%$ probability level and the highest perceived noise level extracted from a CDF at the $97.5 \%$ are chosen (for a significance level of $a=0.05$ ). The use of the P-Box uncertainty approach is conservative, but it is statistically justifiable for the given inputs to the simulations. Note that the choice of significance level is somewhat subjective and can alter the findings.

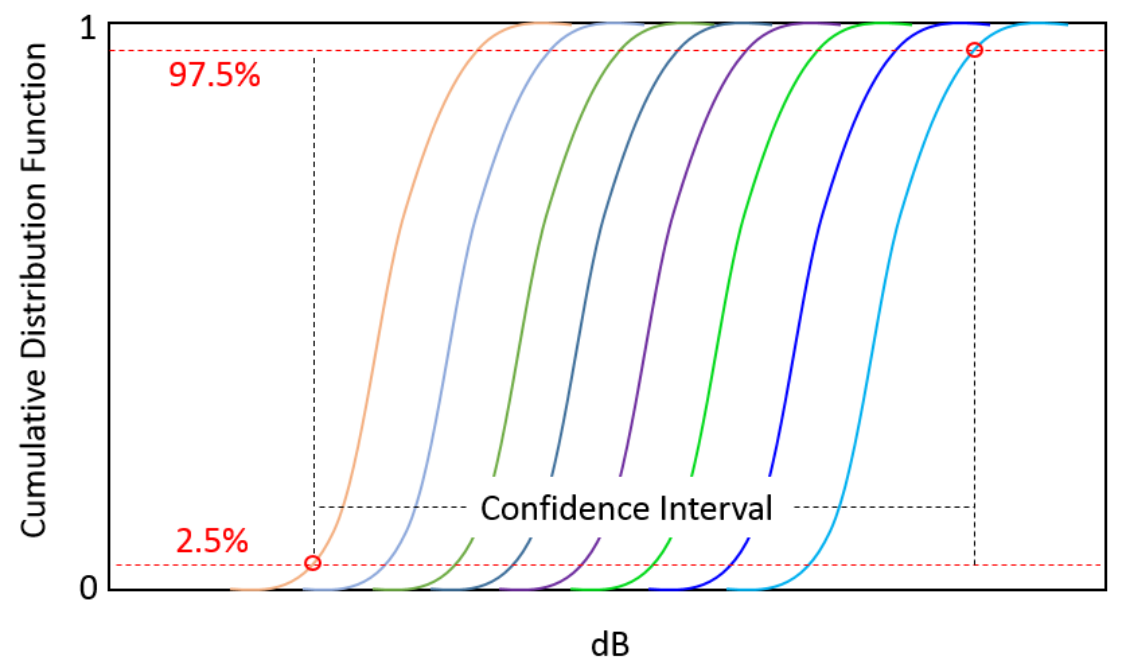

Figure 3: Example signature P-Box.

\section{B. Point-Collocation Non-intrusive Polynomial Chaos}

A second method utilized in this research was non-intrusive polynomial chaos with point-collocation. The polynomial chaos method has been demonstrated as a viable and economical means of uncertainty quantification, as compared to traditional sampling methods such as Monte Carlo, for CFD-based applications. ${ }^{12}$ Polynomial chaos is a surrogate modeling technique based on a spectral representation of uncertainty. An important aspect of spectral representation of uncertainty is that a response value or random function, $F$, can be decomposed into separable deterministic and stochastic components, as shown in Eq. 1.

$$
F(\boldsymbol{d}, \boldsymbol{\xi}) \approx \sum_{i=0}^{P} A_{i}(\boldsymbol{d}) \Psi_{i}(\boldsymbol{\xi})
$$

Here, $A_{i}$ is the deterministic component and $\Psi_{i}$ is the random variable basis functions corresponding to the $i^{\text {th }}$ mode. The basis functions, $\Psi_{i}$, of each random variable are determined using the Askey key ${ }^{13}$ and are dependent on the distribution of each random variable. The response $F$, is a function of independent, random variables, $\boldsymbol{\xi}$, and deterministic variables, $\boldsymbol{d}$. This series is in theory an infinite series but is truncated in practice. To form a complete basis or for a total order expansion, $N_{t}$ terms are required, which can be computed from Eq. 2 for a polynomial chaos expansion (PCE) of order $p$ and a number of random dimensions or variables, $n$.

$$
N_{t}=P+1=\frac{(n+p) !}{n ! p !}
$$

Further details on polynomial chaos theory are given by Ghanem ${ }^{14}$ and Eldred. ${ }^{15}$

To compute the expansion coefficients, $A_{i}$, the well-known non-intrusive point collocation method is utilized. ${ }^{12}$ The response, $F$, is sampled at locations throughout the random variable space, and the expansion coefficients may be computed with an over-determined, least squares approach. At least $N_{t}$ samples are needed for this procedure; Hosder et al. recommend an oversampling ratio of two (i.e., $2 \cdot N_{t}$ samples). Note that a Latin hypercube sampling approach is used to ensure coverage of the domain spanned by the random 
variables. Once the PCE expression in Eq. 1 is obtained, analytical expressions for the mean and standard deviation of $F$ are available, ${ }^{15}$ as are Sobol indices (global nonlinear sensitivity parameters). ${ }^{16}$ Alternatively, Monte Carlo sampling of the low-cost PCE may be conducted in order to obtain probability density functions (PDF), higher order moments, probabilities of failure, etc.

\section{Monte Carlo Variance Decomposition}

Monte Carlo simulations have been utilized to characterize uncertainty in stochastic systems for many years and can provide robust estimates of uncertainty for low computational cost simulations. Although the necessary runs for Monte Carlo simulations are independent from the number of uncertain factors, variance decomposition from Monte Carlo simulations suffers from the "curse of dimensionality". The total number of runs required to estimate only the first order terms (no interactions) is equal to $m(k+1)$ where $k$ is the number of uncertain parameters and $m$ is the number of runs necessary for convergence of the original simulation. To estimate the interactions of the first order terms, the total run count scales with $2^{k}$. Thus, this methodology is only applicable in scenarios where computational cost is extremely low. Other variance based methods such as the Fourier amplitude sensitivity test (FAST) ${ }^{18,17}$ and high dimensional model representation (HDMR) ${ }^{19}$ received consideration for use in this research. However, the relative ease of implementation of Monte Carlo based Sobol indices and low computational cost of the atmospheric propagation prevented the exploration of more complex methods. As the number of disciplines and fidelity of low-boom UQ research increase, more sophisticated sensitivity methods will need to be utilized. An exploration of non-surrogate based methodologies will be a subject of future work.

The methodology behind Monte Carlo variance decomposition is to run successive Monte Carlo simulations on different sample sets and isolate the variance in the output attributable to a specific factor, or interactions of factors. For the Sobol indices implementation, ${ }^{20,21}$ the factor of interest is "frozen"while other factors are allowed to vary. This amounts to a one factor at a time (OFAT) experimental design. To estimate interactions, the desired interacting factors are frozen while all other factors are allowed to vary. In most engineering applications, the uncertainty attributable to interactions is small and not reported. However, if large interactions are present, the chances of misrepresenting the overall uncertainty are drastically increased. The formulations for estimating the Sobol indices from Monte Carlo variance decomposition are given in Eq. 3 through 7.

$$
\begin{gathered}
f_{o} \approx 1 / N \sum_{j=1}^{N} f\left(\mathbf{X}_{\mathbf{1}_{\mathbf{j}}}\right) \\
D \approx 1 / N \sum_{j=1}^{N} f^{2}\left(\mathbf{X}_{\mathbf{1}_{\mathbf{j}}}\right)-f_{o}^{2} \\
D_{i} \approx 1 / N \sum_{j=1}^{N} f\left(\mathbf{X}_{\mathbf{1}_{\mathbf{j}}}\right) f\left(\mathbf{X}_{\mathbf{i}_{\mathbf{j}}}\right)-f_{o}^{2}
\end{gathered}
$$

Here, the matrix $\mathbf{X}_{\mathbf{1}_{\mathbf{j}}}$ is the primary design matrix where all factors are allowed to vary and $\mathbf{X}_{\mathbf{i}_{\mathbf{j}}}$ is the $i^{\text {th }}$ design matrix which holds constant the $i^{\text {th }}$ factor. The total variance assuming no interactions is given by Eq. 4. The corrected total variance, $D_{T}$, given by Eq. 6, is needed to attribute the percentage of variance to each factor taking into account the "double book-keeping" of the possible interaction of terms $\left(D_{i}\right.$ is the principle component of variance attributable to factor $i$ which includes all interactions).

$$
D_{T}=\sum_{i=1}^{N} D_{i}
$$

The conditional or "leftover" variance when a single factor is held constant and all other factors are varied is attributable to all other varying factors. Therefore, the variance attributable to the factor held constant is proportional to the inverse of the conditional variance.

$$
S_{i} \approx \frac{1}{D_{T}}\left(\frac{D-D_{i}}{D}\right)
$$


Eq. 7 gives the formulation for the Sobol index for the $i^{\text {th }}$ factor which represents a scaled (0 to 1 ) representation of the overall uncertainty in the process attributable to factor $i$.

\section{Computational Setup}

This section will detail the framework for conducting this research and the airframe model utilized as a case study. The type of analysis being performed required a robust environment and integration of multidisciplinary simulations including fluid-structure interactions. The system architecture developed for this research was designed to allow for future investigation of other flight concepts regardless of vehicle configuration.

\section{A. Airframe Model}

The airframe model that was utilized as a case study in this research is a supersonic aircraft designed as a proof of concept for future full-scale low-boom commercial supersonic vehicles. The concept vehicle has a wing span of approximately $30 \mathrm{ft}$ and a reference body length of approximately $90 \mathrm{ft}$. The design of the vehicle has been altered to allow an unpowered flight condition to studied; the powered inlet and exhaust engine faces were replaced with a flow-through nacelle. This was done in part to decrease computational resources and aid in convergence of the results. Future work will be dedicated to integrating powered conditions into this analysis. A solid model of the airframe is shown in Figure 4.

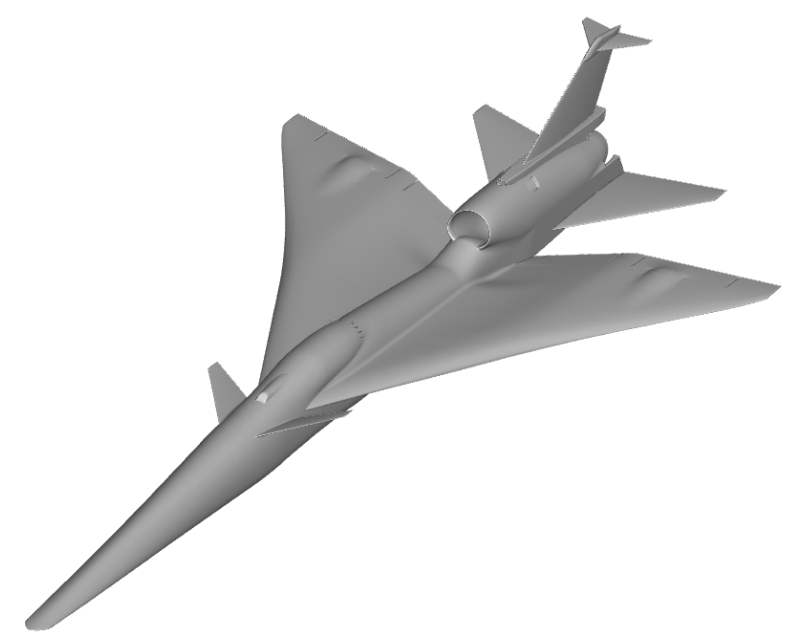

Figure 4: Airframe model.

\section{B. Simulation Procedures}

The Cart3D ${ }^{22,23}$ CFD package was utilized for this research due to its established history of prediction of near field sonic boom pressure waveforms and its wide use across NASA. Due to the number of CFD runs to be completed, an inviscid analysis package was utilized to reduce overall computational resources. In low boom vehicle design, effects of boundary layer and shock wave interaction are modeled and utilized to shape the overall signature in fully viscous solvers. Employing an inviscid solver will not capture these physics; however, multifidelity methods ${ }^{24}$ can be employed once sufficient inviscid solver runs have been completed. The multifidelity methods for this research are left to future work. The finite element analysis (FEA) package utilized to determine the static aeroelastic deformation was MSC NASTRAN. ${ }^{25}$ A high fidelity finite element model (FEM) developed for use in structural sizing of the concept vehicle was used for the structural analysis. A linear static solution with inertial relief was employed. The computational flow chart for generating the near field pressure waveforms is shown in Figure 5. First, a set of deterministic flight conditions consisting of angle of attack and Mach number is prescribed. Then, the deterministic conditions are perturbed such that the surrogate model can be constructed. Note that the CFD domain and adjoints are different in 
this portion of the procedure as no off-body signatures are being sought, only the pressure distribution on the body is of importance here. Next, to transfer the extracted surface pressure distribution, $\boldsymbol{P}$, from the CFD mesh to the FEM mesh, the Discrete Data Transfer Between Dissimilar Meshes (DDTBDM) ${ }^{26}$ is used. Then the transfered pressure distribution, $\boldsymbol{P}^{\prime}$, is used as the loading condition for the FEA. A set of uncertain structural parameters are initialized for each run and the static aeroelastic deformation is obtained, represented here by $\vec{X}$.

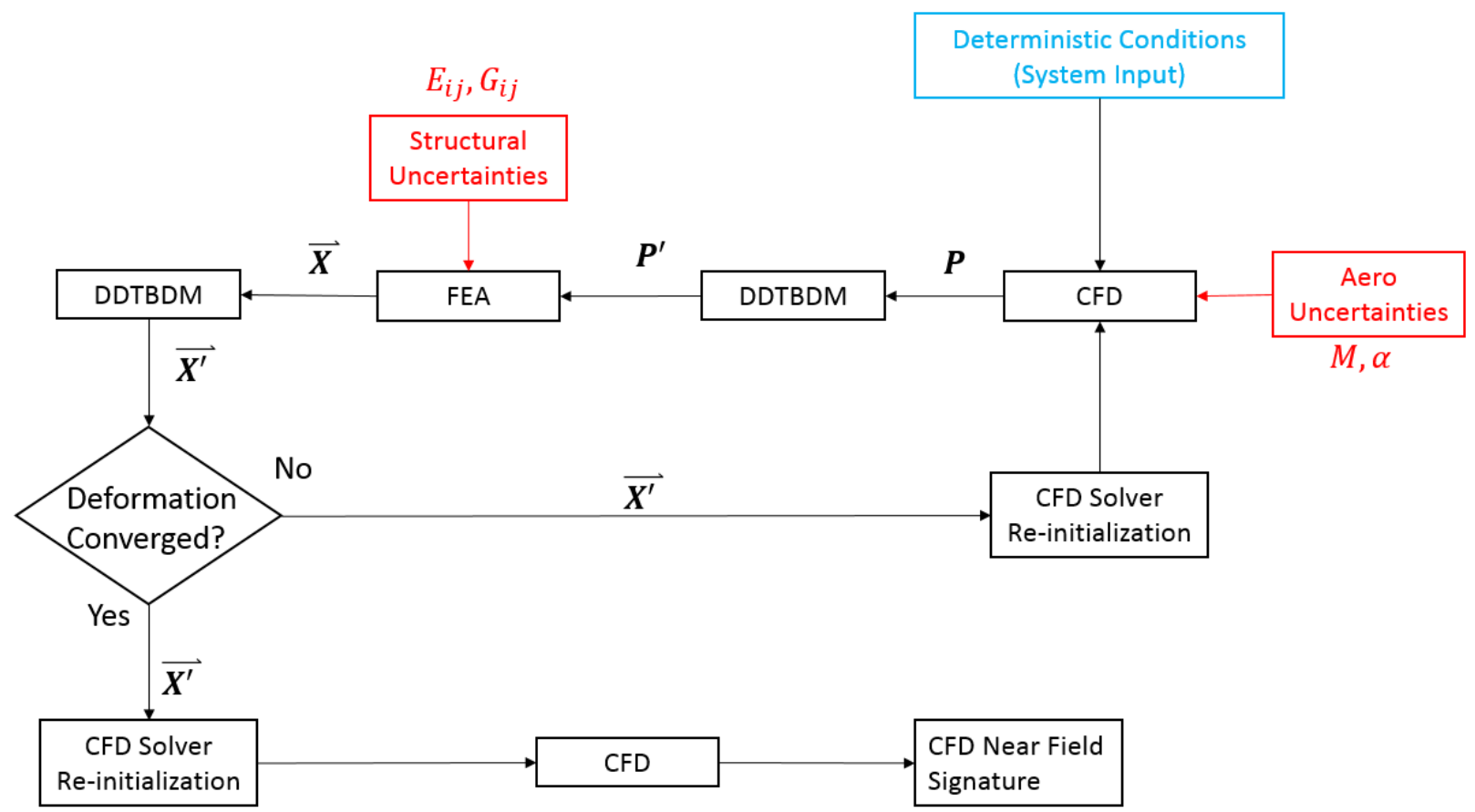

Figure 5: Near field computational flowchart.

The CFD and FEM surface meshes are shown in Figure 6 to illustrate the complexity of the procedure to transfer the loading between models. The CFD surface mesh consists of approximately 240,000 nodes, whereas the FEM surface mesh contains approximately 30,000 nodes. In regions of the model with large geometric curvature, such as the wing leading edge, the CFD mesh contains orders of magnitude more nodes.

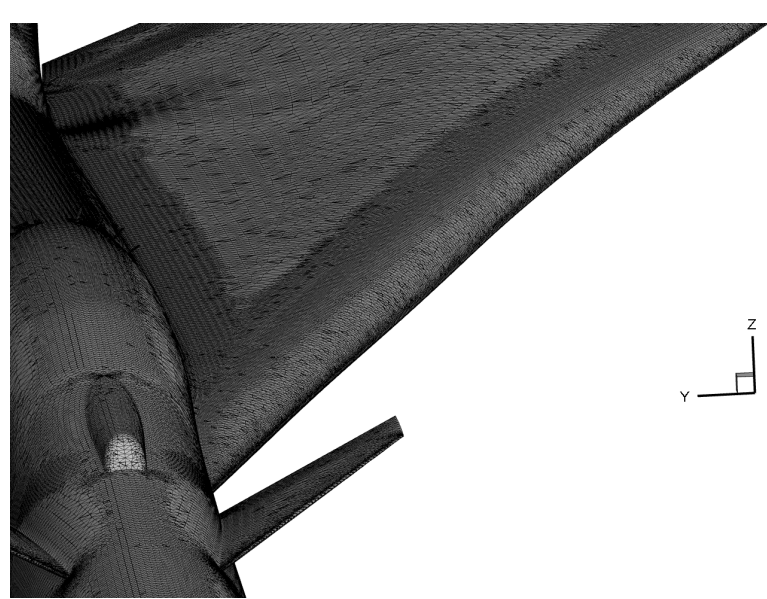

(a) CFD surface mesh.

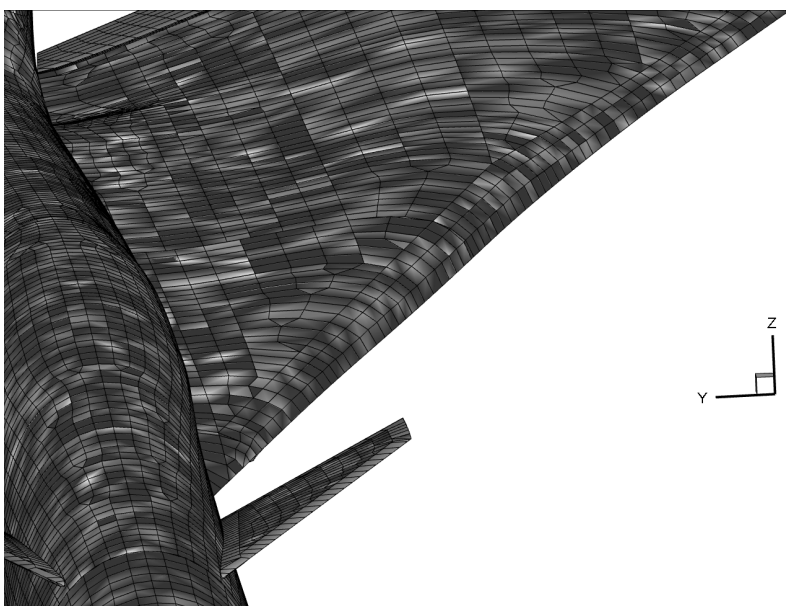

(b) FEM surface mesh.

Figure 6: Comparison of CFD and FEM surface meshes. 
The last three steps are performed in an iterative procedure until the static aeroelastic deformation has converged. With each new iteration, the deformed OML is generated and passed back to the CFD solver. This procedure ensures that the static aeroelastic deformation used to generate the near field pressure wave form is an accurate representation of expected deformation. Once a converged deformation has been obtained, the CFD solver is re-initialized to obtain the near field pressure wave form. The entire procedure is repeated for the number of runs, $N_{T}$, necessary to construct the surrogate model (refer to Eq. 2).

Once the post-processed near field pressure waveforms are generated for each flight condition, the pressure waveforms are passed to an atmospheric propagation code, sBOOM. ${ }^{2}$ The propagation code simulates how the near field pressure waveform will change while passing through the atmosphere to the ground. The sBOOM propagation code produced ground signatures (overpressure vs. time) and performed an integration to produce the A-weighted sound exposure level (ASEL). The ground signature was then passed to the loudness code, ${ }^{27}$ which computed perceived loudness, PLdB, and C-weighted sound exposure level, CSEL. The transfer method of the near field waveform to the atmospheric propagation code depends on the particular UQ method and model. The details are given in the following section.

\section{Uncertainty Modeling}

This section will detail the uncertain parameters investigated in this work. In addition, the sequential process of uncertainty modeling, the specific surrogate models assembled and the estimates of uncertainty they generate, will be introduced.

\section{A. Uncertain Parameters}

The CFD analysis, FEM, and the atmospheric propagation all have uncertain factors which impact the overall prediction of noise metrics at the ground. In the near field, which includes the coupled CFD and FEA, eight uncertain factors were studied and are listed in Table 1. Each of these factors was characterized as an aleatory uncertainty and assigned a normal distribution. The standard deviations for the Mach number and angle of attack were selected to align with previous near field UQ. ${ }^{5}$ The standard deviations for the structural parameters were also chosen to align with previous UQ research. ${ }^{9}$ Note the six uncertain structural parameters were down selected from an original set of 55 parameters. Details of this screening analysis are given in section V. General descriptions of the uncertain structural parameters are listed below. The locations of the parameters are given in Figure 7.

- $S_{1}$ is the modulus of elasticity of the isotropic material for the engine deck and supporting structure.

- $S_{2}$ is the modulus of elasticity of the isotropic material for the structures that include the majority of the internal structure of the model, which incorporates the ribs, spars, bulkheads, and stringers.

- $S_{3}$ is the modulus of elasticity of the isotropic material for the structures that encompass the skin on the wing leading edges, cabin, fuselage, vertical tail, horizontal tail, and t-tail.

- $S_{4}$ is the modulus of elasticity of the isotropic material for the elements utilized to transfer loading from the canards, horizontal tail, and t-tail to the fuselage.

- $S_{5}$ is the shear modulus of the isotropic material for the elements utilized to transfer loading from the canards, horizontal tail, and t-tail to the fuselage.

- $S_{6}$ is the modulus of elasticity of the composite material in the fiber direction of the structure that includes the skin on the wing not located on the leading edge

Note that model structures incorporating parameters $S_{4}$ and $S_{5}$ exist to simplify the transfer of the loading from external surfaces (canard, tails) to the fuselage. In a higher fidelity structural model, these parameters would more accurately model the load path. $S_{4}$ and $S_{5}$ could be considered a source of model form or epistemic uncertainty in a multifidelity analysis. In Figure 7, the size of model structures incorporating parameters $S_{4}$ and $S_{5}$ have been significantly increased to illustrate their locations within the model.

Six uncertain factors in the atmospheric propagation were studied. Four of the six factors in the atmospheric propagation, shown in Table 2, were characterized as aleatory uncertainties and assigned Gaussian distributions. 
Table 1: Near field CFD and structural aleatory input parameters.

\begin{tabular}{cccc}
\hline \hline Input & Distribution & Mean & Coef. of Var. \\
\hline Angle of Attack (Deg.) & Gaussian & 1.7 & $5 \%$ \\
Mach Number & Gaussian & 1.42 & $0.15 \%$ \\
$S_{1}(\mathrm{ksi})$ & Gaussian & 16000 & $4 \%$ \\
$S_{2}(\mathrm{ksi})$ & Gaussian & 10300 & $4 \%$ \\
$S_{3}(\mathrm{ksi})$ & Gaussian & 10500 & $4 \%$ \\
$S_{4}(\mathrm{ksi})$ & Gaussian & 28300 & $4 \%$ \\
$S_{5}(\mathrm{ksi})$ & Gaussian & 11055 & $4 \%$ \\
$S_{6}(\mathrm{ksi})$ & Gaussian & 21800 & $4 \%$ \\
\hline \hline
\end{tabular}

(b) $S_{2}$.

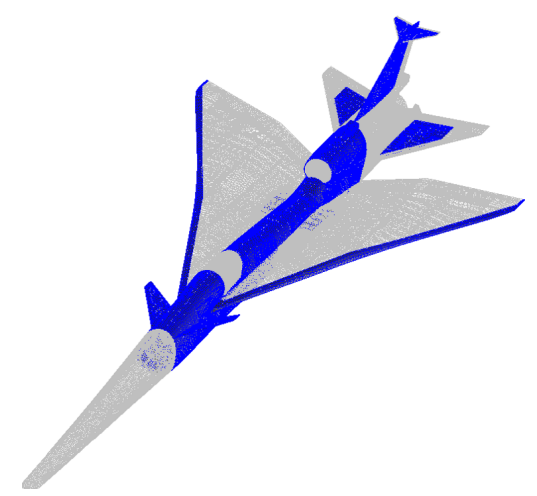

(c) $S_{3}$.

(a) $S_{1}$.

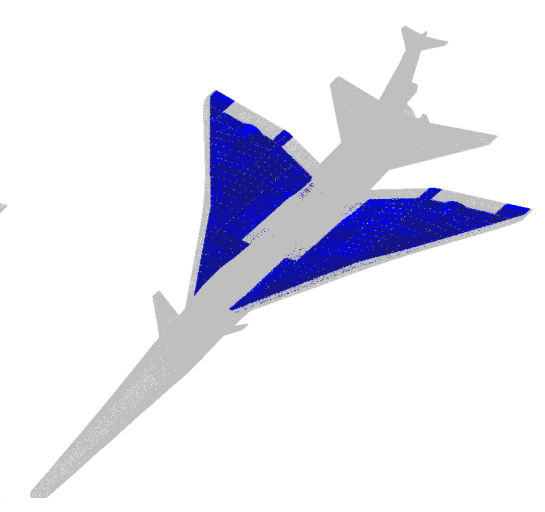

(d) $S_{4}$.

(e) $S_{5}$.

Figure 7: Locations of structural parameters

Table 2: Atmospheric propagation aleatory input parameters.

\begin{tabular}{cccc}
\hline \hline Input & Distribution & Mean & Std. Dev. \\
\hline Relative Humidity $(\mathrm{RH})$ & Gaussian & Altitude Dependent & $1 \%$ \\
Temperature & Gaussian & Altitude Dependent & $1 \%$ \\
X-wind & Gaussian & Altitude Dependent & $13.12 \mathrm{ft} / \mathrm{s}$ \\
Y-wind & Gaussian & Altitude Dependent & $13.12 \mathrm{ft} / \mathrm{s}$ \\
\hline \hline
\end{tabular}


Note that the mean value for each of the parameters in Table 2 is altitude dependent. The relative humidity, temperature, $\mathrm{X}$-wind, and Y-wind are represented as deviations from the deterministic profiles and are implemented as a pure bias factor. The wind direction convention for this application is wind in the (positive) X-direction is a tailwind and wind in the Y-direction is cross wind. The uncertainty in the wind profile was determined from information compiled by Schwartz, ${ }^{28}$ which includes measurement error, as well as temporal and spatial variances. To assess the changes in sensitivities and overall confidence intervals with changes in atmospheric profiles, two deterministic profiles were studied. The first deterministic profile was the standard atmospheric profile, ${ }^{29,30}$ which contained no atmospheric winds. The second deterministic atmospheric profile was adopted from the second sonic boom prediction workshop ${ }^{31}$ for a case from Green Bay, WI during the winter of 2013. The Green Bay deterministic atmospheric profile is shown in Figure 8; the standard atmospheric deterministic profile is shown in Figure 9.
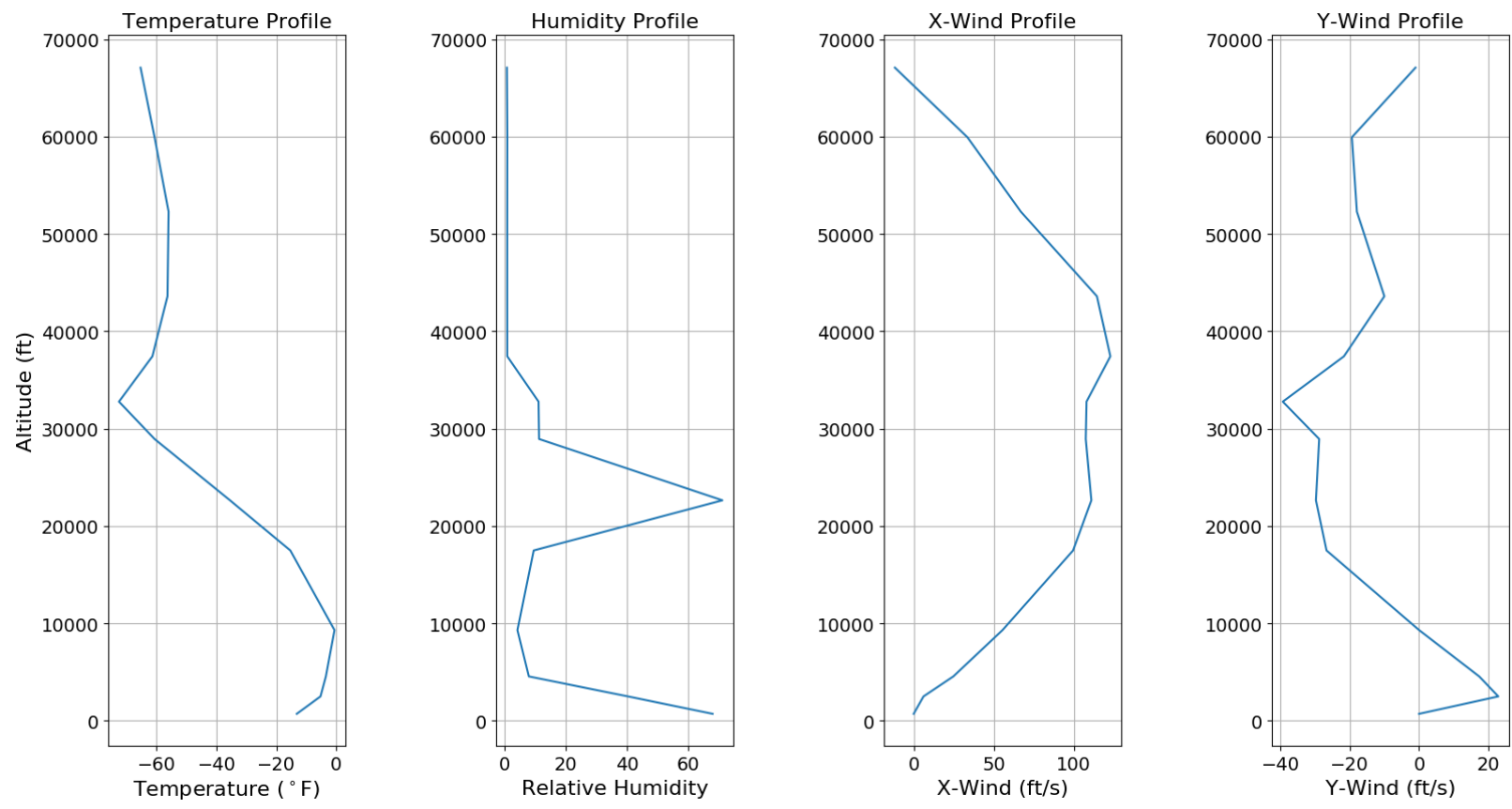

Figure 8: Green Bay atmosphere deterministic profiles.

Two of the six uncertain factors in the atmospheric propagation were treated as epistemic uncertainties and are given in Table 3. There is no inference made about the distribution of epistemic parameters, only a bounding interval is given. The intervals for these factors were chosen to align with previous low-boom UQ work. ${ }^{5}$ For the airframe model being investigated, Phillips and West ${ }^{8}$ previously found that uncertainty in ground altitude was the major contributor to overall variance in the ground noise response (for ASEL and PLdB). Therefore, a smaller epistemic interval for ground altitude simulating a known flight path will also be considered.

Table 3: Atmospheric propagation epistemic input parameters.

\begin{tabular}{cc}
\hline \hline Input & Interval \\
\hline Ground Altitude & {$[0,5000 \mathrm{ft}]$ or $[600,1400 \mathrm{ft}]$} \\
Reflection Factor $(\mathrm{RF})$ & {$[1.8,2.0]$} \\
\hline \hline
\end{tabular}

\section{B. Modeling Procedures}

A single set of near field deterministic conditions, given from the mean values in Table 1, and three atmospheric deterministic conditions were studied. The three atmospheric profile combinations are shown in 

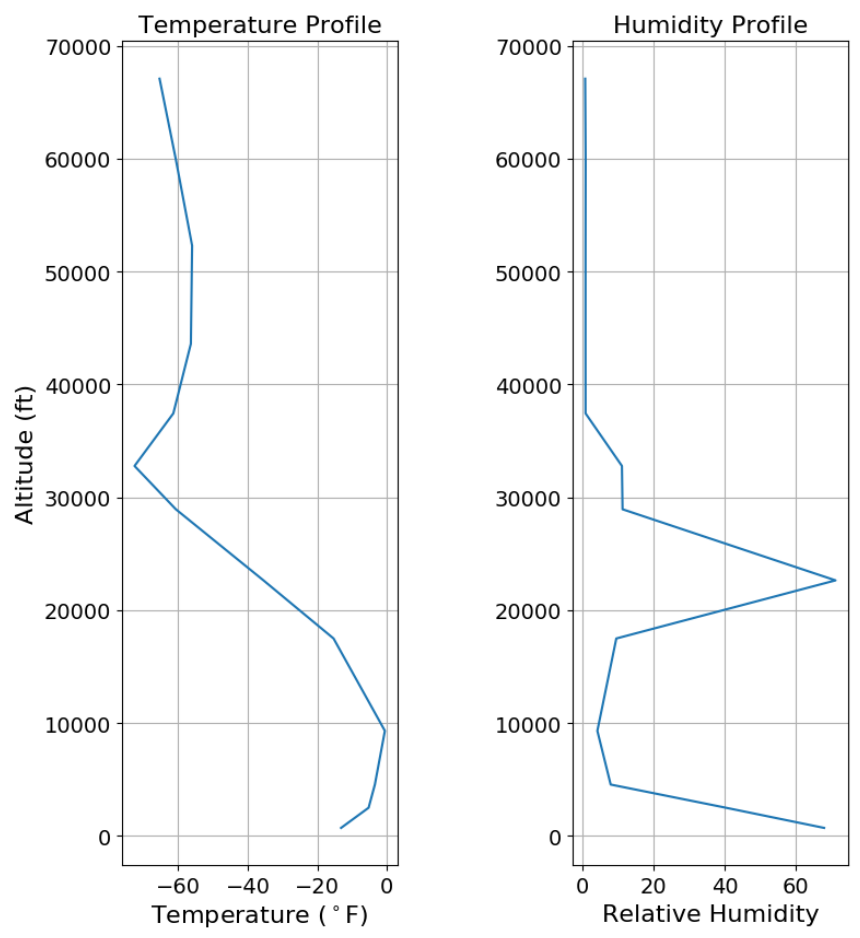

Figure 9: Standard atmosphere deterministic profiles.

Table 4. The choice of three atmospheric profile combinations allows for comparisons of sensitivities across profiles, as well as ground altitude interval selections.

Table 4: Atmosphere profile combinations studied.

\begin{tabular}{cc}
\hline \hline Atmospheric Profile & Ground Altitude Interval (ft) \\
\hline Standard & {$[0,5000 \mathrm{ft}]$} \\
Green Bay & {$[0,5000 \mathrm{ft}]$} \\
Green Bay & {$[600 \mathrm{ft}, 1400 \mathrm{ft}]$} \\
\hline \hline
\end{tabular}

For the near field uncertain parameters, a polynomial chaos model was generated with an oversampling ratio of two giving a run count of 90 per iteration (refer to Eq. 2). Including the fluid-structure interaction (FSI) convergence runs, this resulted in 360 CFD, 180 FEA, and 360 associated DDTBDM runs. The samples for the PCE models were generated using a Latin hypercube sampling routine with the distributions given in the previous section (refer back to Table 1). The response modeled for these PCEs was the ground noise when propagated through a deterministic atmosphere. This allowed the relative uncertainties of all near field factors to be assessed. A second set of PCE models was generated from the near field data by using the local pressure along the near field pressure waveform as the response. From these local polynomial chaos models, estimates for both the local Sobol indices and the local confidence intervals can be generated. Once the confidence interval was established, Fourier dispersions ${ }^{6,7}$ were generated to pass to the atmospheric propagation. This methodology effectively decouples the atmospheric propagation from the near field simulation from a UQ perspective. An advantage to this approach is that the overall number of CFD runs can be reduced, allowing more near field conditions to be studied for the same computational cost. The drawback is that the uncertainty represented by the near field factors is only discernible as a combined effect at the ground and cannot be assigned to individual factors. The reader is referred to Phillips and West ${ }^{8}$ for a more detailed discussion.

When the data from the near field had been transfered to the propagation codes, a Monte Carlo variance decomposition analysis was performed. This process produced tens of thousands of runs where the response 
was the loudness at the ground. Employing the methodology outlined in section II, the global uncertainties were estimated for the atmospheric factors and the bulk near field. A second analysis of Monte Carlo variance decomposition was performed to calculate the local uncertainties. Similar to the near field local PCE models, the response for the analysis was the local pressure along the ground signature. A flow chart of the uncertainty modeling process after near a field CFD pressure waveform is obtained is shown in Figure 10.

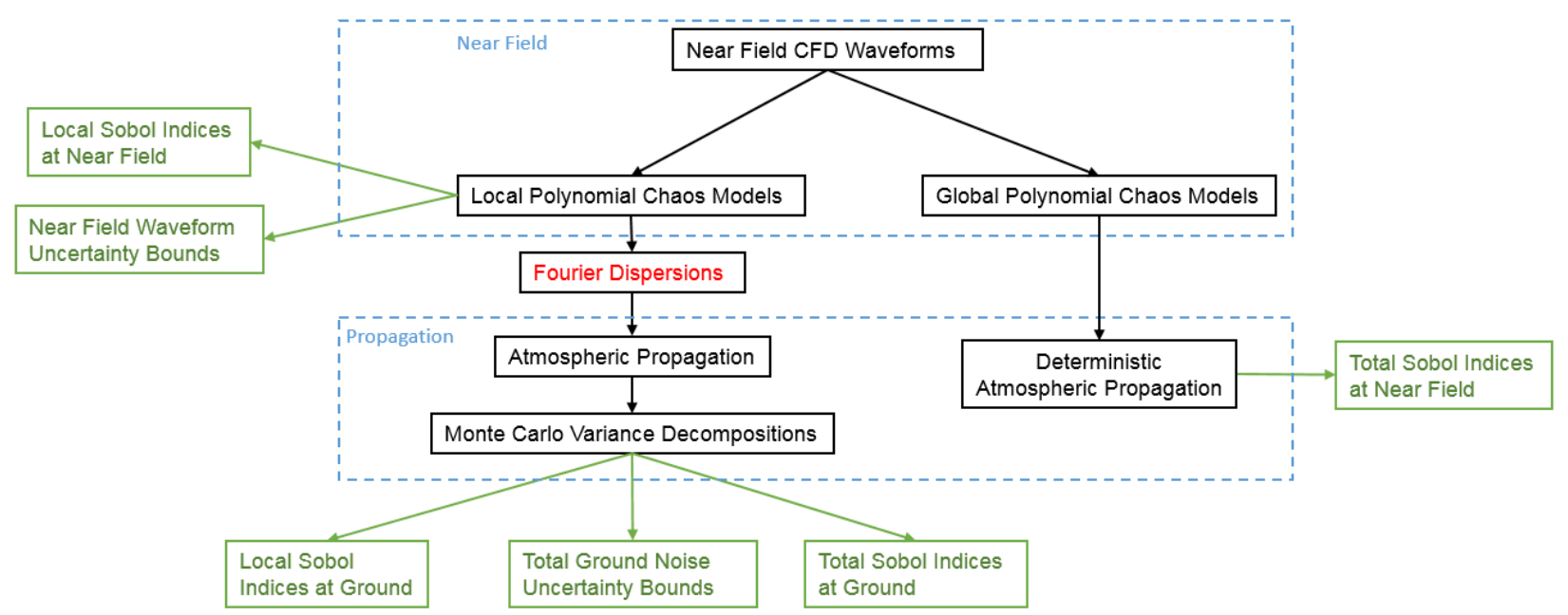

Figure 10: Uncertainty modeling flow chart.

\section{Results}

First, results of the structural parameter screening analysis will be presented as a means to reduce the dimensionality of the ground noise surrogate model. Then, the results from the iterative fluid-structure interaction will be given. Next, a comparison of the results for baseline, undeformed configuration, and the static aeroelastic deformed configuration including offtrack analysis, will be shown for both the standard atmosphere and the Green Bay atmospheric profile containing winds. Then, results from the global and local near field PCE models for the deterministic atmospheric combinations will be presented. Finally, the local and global Monte Carlo variance decomposition analyses for the uncertain atmospheres will be detailed. All of the results in this section were generated for a nominal Mach number of 1.42 and an angle of attack of 1.7 to align with previous work on this configuration. ${ }^{8}$

\section{A. Structural Parameter Screening Analysis}

Due to the inherent coupled nature of the fluid-structure interaction, structural parameters are inseparable from near field CFD uncertain parameters. The original set of uncertain structural parameters included moduli for each material used in the FEM. To reduce the overall number of uncertain parameters, a screening analysis was performed on the 55 uncertain structural parameters to identify those with the largest overall sensitivities. To perform this analysis, the deterministic surface pressure distribution was first generated from CFD. Next, multiple FEA were completed varying the uncertain structural parameters. Fundamentally, changes in the OML of the vehicle will alter the near field pressure waveform, which in turn has the potential to effect ground noise. As it is computationally infeasible to estimate sensitivities to ground noise of individual nodal displacements, the aggregate nodal displacements of specific nodes were tracked. Five locations on the model were tracked for displacements and used as responses for the sensitivity study, nodal displacements at the nose, canard tip, wing tip, horizontal tail tip and t-tail tip. The aggregate nodal displacements are assumed to be sufficient for estimating structural parameter sensitivities with respect to ground noise. To estimate the structural sensitivities, a polynomial chaos surrogate model was assembled for each of the nodal responses. With 55 uncertain structural parameters, a second order PCE model, twice over sampled, necessitated 3192 runs. An underlying assumption with this screening analysis is that the structural 
parameter sensitivities would not change significantly if small perturbations were made to the surface pressure distribution. The results from the sensitivity analysis are given in Table 5 .

Table 5: Selected displacement sensitivities to uncertain structural parameters.

\begin{tabular}{cccccc}
\hline \hline Structural Parameter & $\delta_{\text {nose }}($ in $)$ & $\delta_{\text {wing }}($ in $)$ & $\delta_{\text {canard }}($ in $)$ & $\delta_{h t}($ in $)$ & $\delta_{t t}($ in) \\
\hline$S_{1}$ & - & - & - & - & $6.6 \%$ \\
$S_{2}$ & $44.4 \%$ & - & $26.9 \%$ & $25 \%$ & $9.3 \%$ \\
$S_{3}$ & $37.2 \%$ & - & $51.1 \%$ & $46.2 \%$ & $61.4 \%$ \\
$S_{4}$ & - & - & $14.1 \%$ & $5.0 \%$ & $4.3 \%$ \\
$S_{5}$ & - & - & - & - & $11.3 \%$ \\
$S_{6}$ & $15.8 \%$ & $95.6 \%$ & $5.7 \%$ & $18.5 \%$ & $3.7 \%$ \\
All Others & $2.6 \%$ & $4.4 \%$ & $2.2 \%$ & $5.3 \%$ & $3.4 \%$ \\
\hline \hline
\end{tabular}

In Table 5, each column represents the relative sensitivities of the uncertain structural parameters with respect to the nodal displacement at the top of that column. For example, structural parameter $S_{6}$ accounts for $95.6 \%$ of the overall variation in the FEM nodal displacement located at the wing tip, $\delta_{\text {wing }}$. Note that only individual sensitivities above $2.5 \%$ are reported; others are considered negligible and listed in Table 5 as "-". FEM nodal displacement distributions for the selected nodes of the model are given in Figure 11. These displacements are results from the Monte Carlo verification of the PCE surrogate model consisting of 15,000 total runs. As expected with a linear static analysis, the displacement response for each of the selected nodes follows a normal distribution.

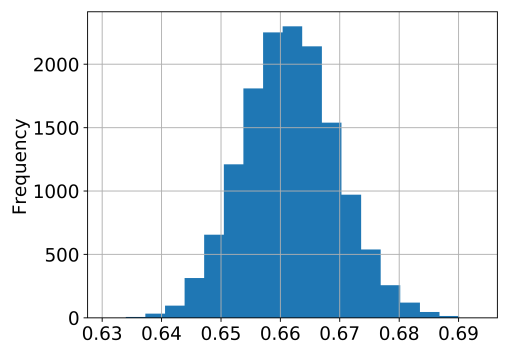

(a) Horizontal Tail

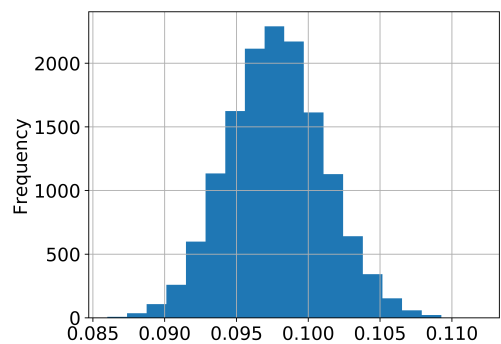

(b) T-Tail

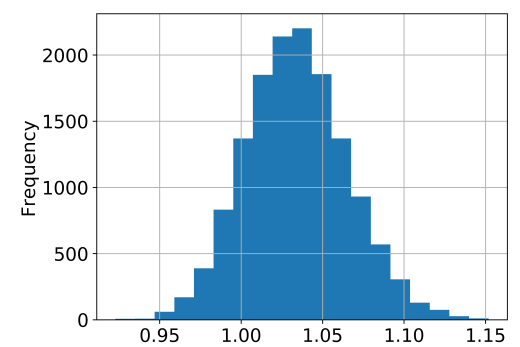

(c) Wing Tip

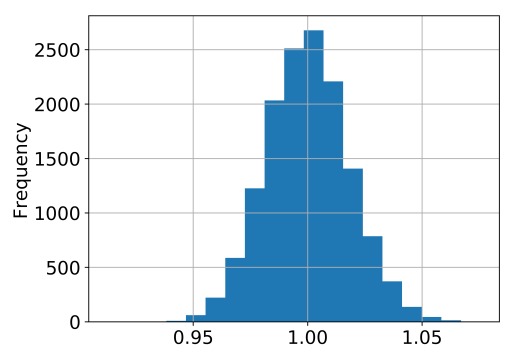

(d) Canard

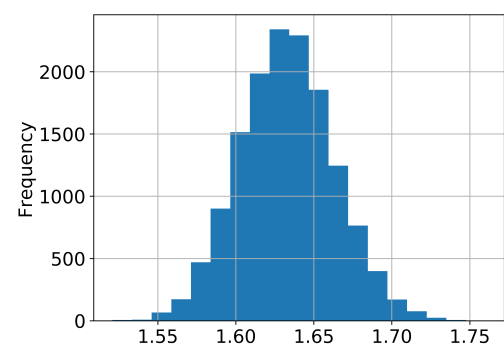

(e) Nose

Figure 11: Selected nodal displacement histograms $H_{T}(\mathrm{a}), T_{T}(\mathrm{~b})$, Wing (c), Canard (d), Nose (e).

\section{B. Fluid-Structure Interaction}

This section will discuss the results for the iterative fluid-structure interaction analysis utilized to converge on the static aeroelastic deformed geometry. An initial CFD run established the baseline $C_{L}$, which was used as the constraint for the analysis. For each iterative cycle through the FEA and CFD, the CFD solver iterated on angle of attack to satisfy the baseline $C_{L}$ constraint. A $0.05 \%$ tolerance of the deterministic $C_{L}$ value was imposed during the iterative procedure. In this analysis, the deformation of the geometry 
necessitated an increase in angle of attack to maintain sufficient lift. The displacements given for each iteration in Table 6 represent the absolute deformation determined from the previous iteration's surface pressure distribution. For example, the displacements for iteration 2 were generated from the CFD pressure distributions of iteration 1 . The displacements for iteration 2 produced the lift coefficient and angle of attack for iteration 2.

Table 6: Convergence of selected FSI parameters with deterministic initial loading.

\begin{tabular}{cccccccc}
\hline \hline Iteration & $C_{L}$ & $\alpha(\mathrm{deg})$ & $\delta_{\text {nose }}($ in $)$ & $\delta_{\text {wing }}($ in $)$ & $\delta_{\text {canard }}($ in $)$ & $\delta_{h t}($ in $)$ & $\delta_{t t}($ in $)$ \\
\hline 1 & 0.098151 & 1.700 & 0 & 0 & 0 & 0 & 0 \\
2 & 0.098132 & 1.929 & 1.4399 & 1.1031 & 0.9397 & 0.8175 & 0.13783 \\
3 & 0.098145 & 1.895 & 1.6429 & 1.0238 & 1.0078 & 0.6418 & 0.09361 \\
4 & 0.098124 & 1.900 & 1.6321 & 1.0354 & 0.9987 & 0.6636 & 0.09829 \\
5 & 0.098192 & 1.900 & 1.6318 & 1.0334 & 1.0001 & 0.6636 & 0.09814 \\
\hline \hline
\end{tabular}

From inspection of Table 6, the nodal displacements have converged to within $1 \%$ and angle of attack has converged to within $0.005^{\circ}$ after the third iteration. Therefore, all subsequent uncertainty analysis runs consisted of three iterations through the FSI procedure. The iterative deformation of the CFD OML from the deterministic loading case is shown for the wing tip in Figure 12 and nose in Figure 13. Iteration 1 represents the original undeformed geometry. All five iterations are plotted; however, after the third iteration, the geometries are indistinguishable from each other. Note that the figures show only a small portion of the model due to the relatively small magnitudes of displacement.

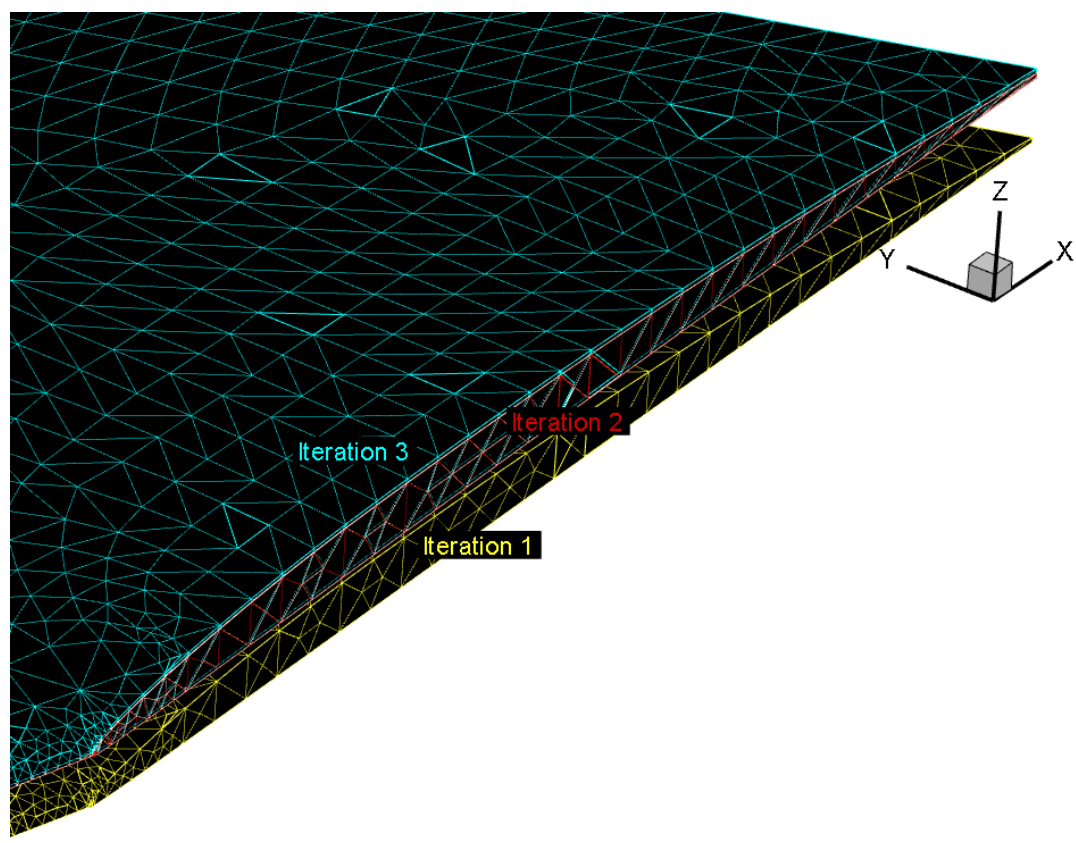

Figure 12: Wing tip deformation convergence.

In addition to the nodal displacements and angle of attack, the near field waveform and ground noise were also tracked through the iterations. The near field waveform convergence for the deterministic case of the FSI procedure is shown in Figure 14 at three body lengths below the model. All subsequent near field waveform plots will also be shown at three body lengths. The near field waveforms after the second iteration do not significantly change and ground noise was converged to 0.05 PLdB after the third iteration. 


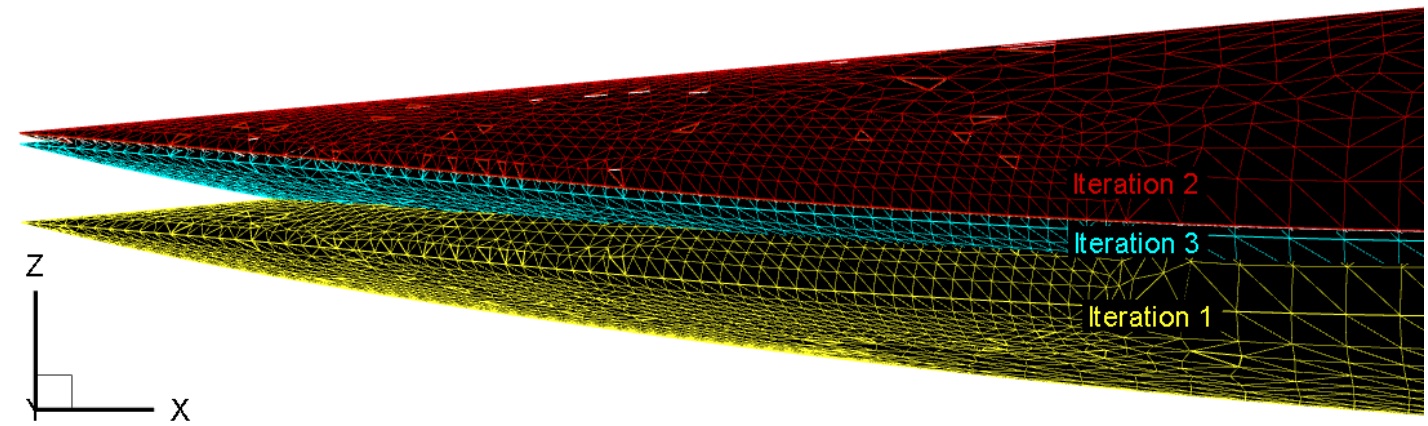

Figure 13: Nose deformation convergence.

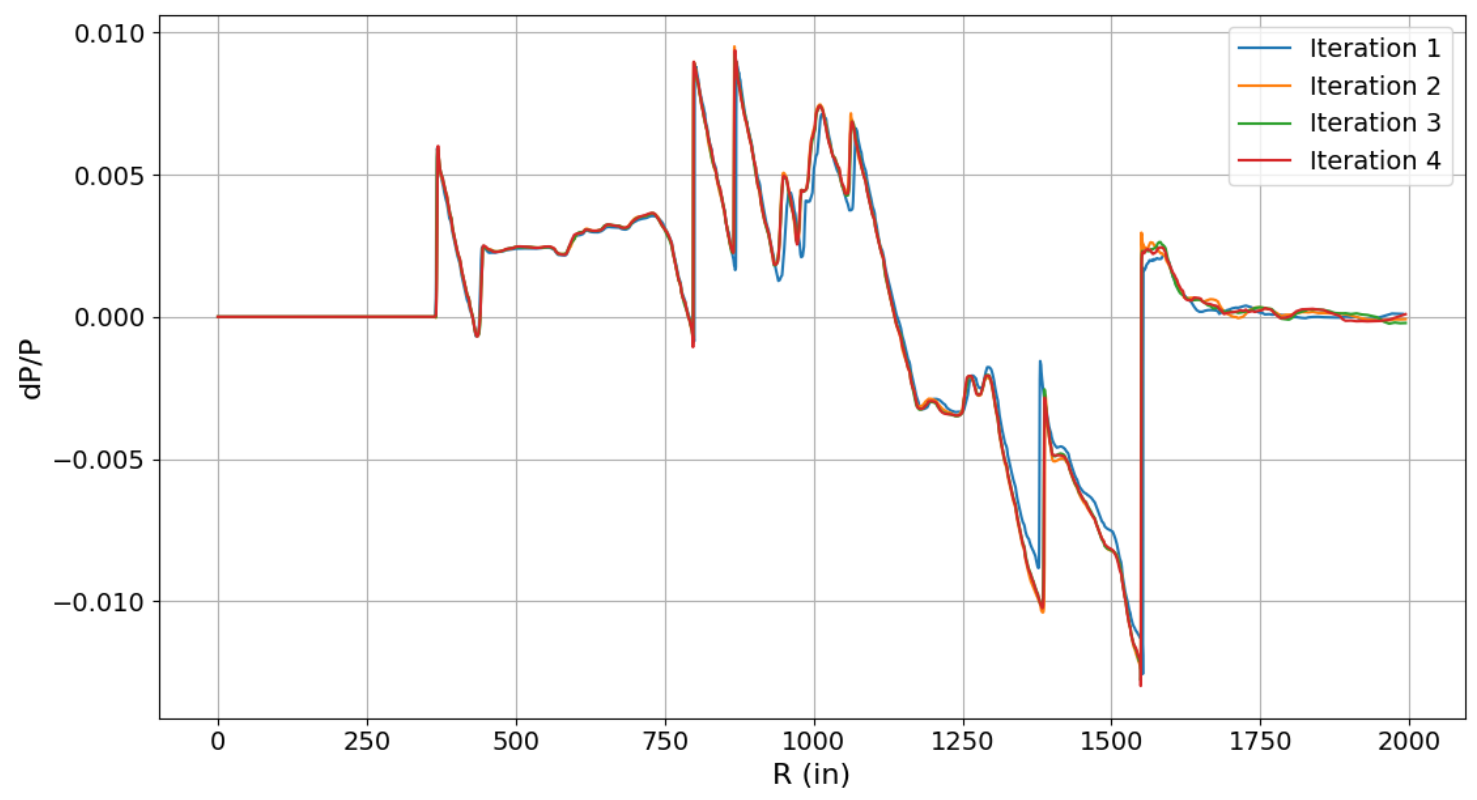

Figure 14: Near field waveform FSI convergence.

\section{Baseline vs. Deformed}

For a comparison between the baseline configuration and the deterministic deformed vehicle, the manufacturer supplied structural parameters were used. To determine ground noise, both the standard atmospheric profile and the Green Bay atmospheric profiles were used. Note, the lateral cutoff angle for the deterministic signature was approximately $45.5^{\circ}$. The near field waveforms at three body lengths for undertrack and offtrack angles are given in Figure 15 for the baseline, undeformed configuration.

The shocks emanating from the nose, canard and wing dissipate significantly with increases in offtrack angle. However, the shocks in the aft portion of the near field waveform remain relatively constant in 


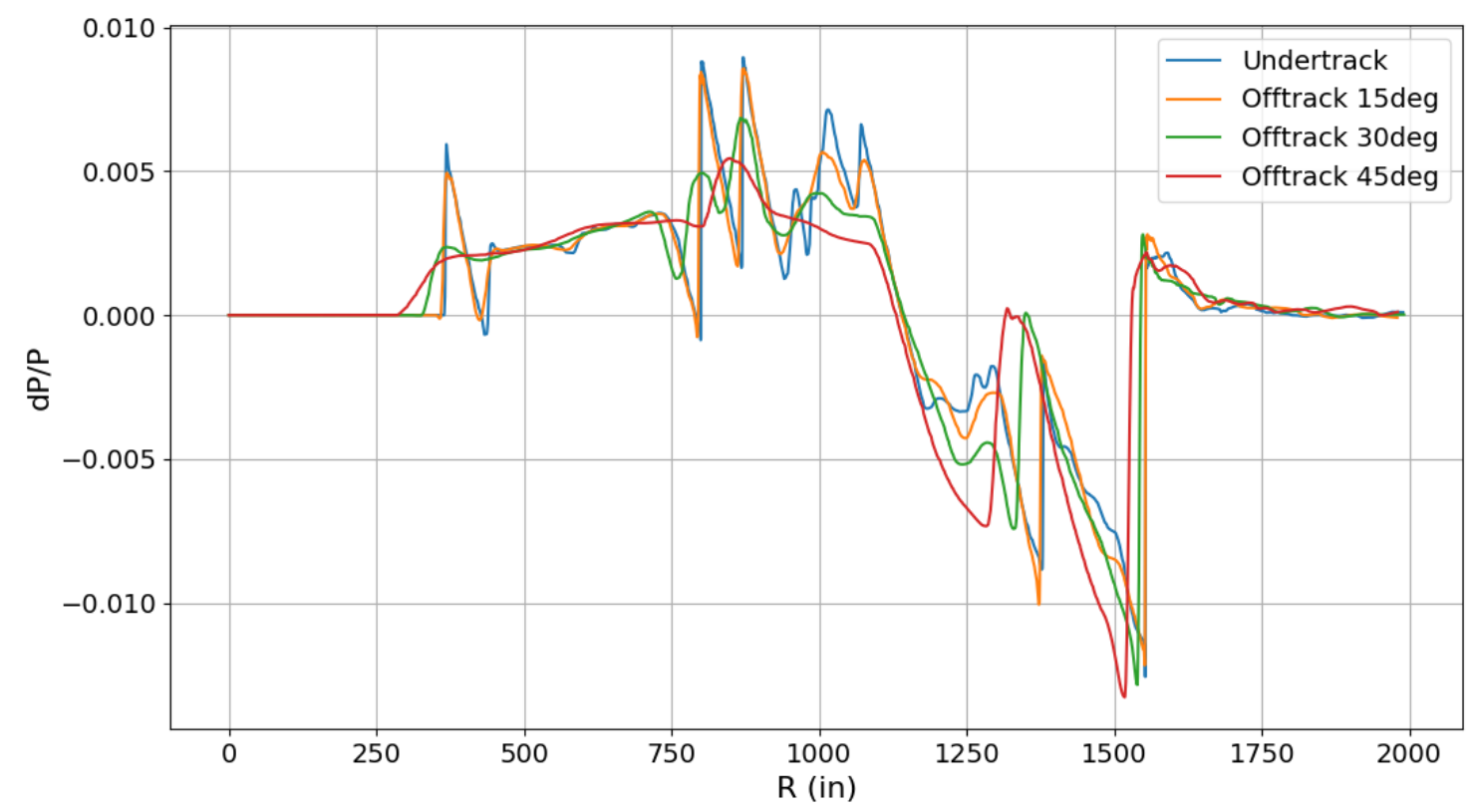

Figure 15: Offtrack waveform comparison.

magnitude. Note the sharp re-compression region in the aft portion of the waveform. In this analysis, the model control surfaces were not deflected to trim the vehicle, which allowed for significant computational savings within the FSI analysis. If the control surfaces are deflected, the aft portion of the near field waveform has a lower magnitude shock structure leading to a lower ground noise. The design point for this concept vehicle to shape the aft end of the near field waveform includes these control surface deflections. Integrating control feedback loops and incorporating control surface deflections into the FSI procedure is left for future work.

Loudness metrics for the standard atmosphere are given in Tables 7 and 8, for the original and deformed configurations, respectively. Notice that for each of the offtrack angles and loudness metrics, the deformed shape was louder. For ASEL, the undertrack loudness increased by about $1.25 \mathrm{dBA}$ with the deformed geometry. Loudness results for the Green Bay atmospheric profile are given in Tables 9 and 10. There is a similar trend to that found for the standard atmosphere in that the loudness is higher for the deformed geometry.

Table 7: Original configuration deterministic standard atmosphere ground loudness metrics.

\begin{tabular}{cccc}
\hline \hline Offtrack Angle & ASEL $(\mathrm{dB})$ & CSEL $(\mathrm{dB})$ & PLdB $(\mathrm{dB})$ \\
\hline $0^{\circ}$ & 72.41 & 92.98 & 86.46 \\
$15^{\circ}$ & 71.68 & 92.53 & 85.42 \\
$30^{\circ}$ & 67.54 & 91.16 & 81.44 \\
$45^{\circ}$ & 60.83 & 88.84 & 75.58 \\
\hline \hline
\end{tabular}

Considering results from both atmospheric profiles, the effect of the static aeroelastic deformation appears to be slightly more significant for the standard atmosphere. However, the baseline ground noise level for the standard atmosphere is approximately 4.5 PLdB louder than the Green Bay ground noise. This illustrates the sensitivity of the ground noise to the atmosphere. Recall that a primary difference between the two atmospheres is the inclusion of both tail and cross winds in the Green Bay profile, which may have a significant impact on the ground noise. These results suggest that the impact of selecting an appropriate atmospheric profile is perhaps more critical than the static aeroelastic analysis. However, this deduction does not speak to the impact of the atmospheric uncertainty relative to the aeroelastic uncertainty for an appropriate atmosphere, which is discussed later. 
Table 8: Deformed configuration deterministic standard atmosphere ground loudness metrics.

\begin{tabular}{cccc}
\hline \hline Offtrack Angle & ASEL $(\mathrm{dB})$ & CSEL $(\mathrm{dB})$ & PLdB $(\mathrm{dB})$ \\
\hline $0^{\circ}$ & 73.65 & 93.52 & 87.47 \\
$15^{\circ}$ & 72.58 & 93.01 & 86.31 \\
$30^{\circ}$ & 67.86 & 92.02 & 81.94 \\
$45^{\circ}$ & 61.36 & 89.46 & 76.11 \\
\hline \hline
\end{tabular}

Table 9: Original configuration deterministic Green Bay atmosphere ground loudness metrics.

\begin{tabular}{cccc}
\hline \hline Offtrack Angle & ASEL $(\mathrm{dB})$ & CSEL $(\mathrm{dB})$ & PLdB $(\mathrm{dB})$ \\
\hline $0^{\circ}$ & 66.08 & 90.78 & 82.00 \\
$15^{\circ}$ & 64.89 & 90.15 & 80.91 \\
$30^{\circ}$ & 60.27 & 88.49 & 76.35 \\
$45^{\circ}$ & 51.94 & 84.56 & 67.92 \\
\hline \hline
\end{tabular}

Table 10: Deformed configuration deterministic Green Bay atmosphere ground loudness metrics.

\begin{tabular}{cccc}
\hline \hline Offtrack Angle & ASEL $(\mathrm{dB})$ & CSEL $(\mathrm{dB})$ & PLdB $(\mathrm{dB})$ \\
\hline $0^{\circ}$ & 66.84 & 91.23 & 82.73 \\
$15^{\circ}$ & 65.46 & 90.70 & 81.49 \\
$30^{\circ}$ & 60.67 & 89.29 & 77.08 \\
$45^{\circ}$ & 52.47 & 85.12 & 68.53 \\
\hline \hline
\end{tabular}

\section{PCE Near field}

The impact of the near field uncertainty was assessed using the polynomial chaos model approach discussed in section II. To assemble a second order PCE model with 8 uncertain factors, 90 runs through the FSI procedure were necessary. The nodal displacement and pressure coefficient statistics, derived from a pure statistics calculation using all 90 runs, is shown in Figure 16. In Figure 16, the mean value is plotted on the left half of the model (positive Y) and the standard deviation is plotted on the right half of the model (negative Y) The statistics for the nodal displacements are as expected in that the wing tip and nose show the most overall displacement and the variance in displacements is higher in the areas of larger displacement. The areas with the largest variance in pressure coefficient values are the canard leading edge, wing leading edge towards the wing tip, and in the area of the reflected nozzle lip shock.

\section{Global PCE Models}

The global PCE models were built for the response of ground noise from a deterministic atmospheric propagation. Both the standard atmosphere and the Green Bay atmosphere were studied along with the three ground noise metrics. The local PCE models used the response of the pressure along the near field waveform to establish confidence intervals. The sensitivity analysis from the global PCE models for the Green Bay deterministic atmospheric profile is given in Table 11.

No individual structural uncertain parameter was significant in the Green Bay deterministic atmospheric propagation. However multiple interactions of structural parameters and structural parameters with CFD uncertain parameters were present in small percentages. For the responses of ASEL and PLdB, many interactions of small significance $(1-2 \%)$ were present, leading to the "All Others" row to having a large 


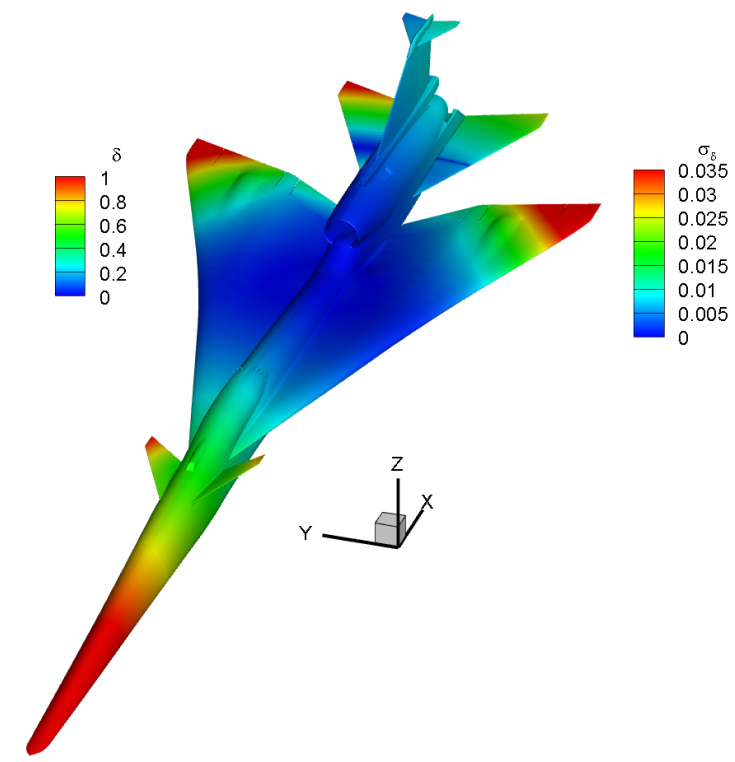

(a) Displacement statistics.

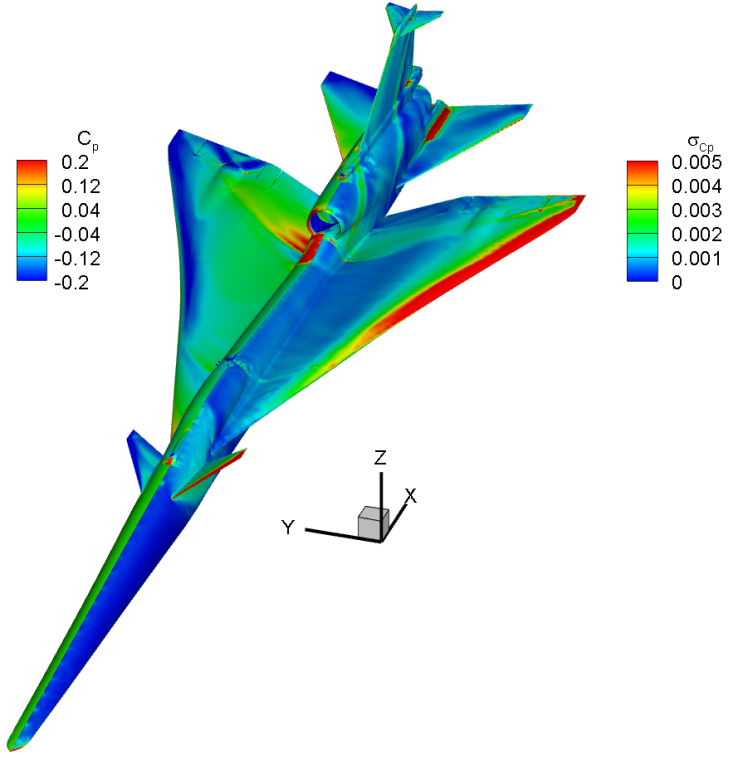

(b) $C_{P}$ statistics.

Figure 16: Surface plot statistics.

Table 11: Expanded near field sensitivities for Green Bay atmosphere deterministic propagation.

\begin{tabular}{cccc}
\hline \hline Uncertain Parameters & ASEL & CSEL & PLdB \\
\hline$S_{1} S_{6}$ & $4.4 \%$ & - & $2.9 \%$ \\
$S_{3} S_{4}$ & $2.9 \%$ & - & - \\
$S_{4} S_{5}$ & - & - & $2.6 \%$ \\
$S_{4} S_{8}$ & $2.9 \%$ & - & - \\
$S_{5} S_{7}$ & $2.9 \%$ & - & - \\
$S_{5} S_{8}$ & $4.3 \%$ & - & - \\
Mach & - & $2.6 \%$ & - \\
$\alpha$ & $54.5 \%$ & $92.9 \%$ & $67.5 \%$ \\
All Others & $28.2 \%$ & $4.5 \%$ & $27.0 \%$ \\
\hline \hline
\end{tabular}

percentage of the overall variance. Variation in CSEL is dominated by the uncertainty in angle of attack. This is likely due to the larger influence of lower frequency content on the CSEL noise metric.

Since interpreting the meaning of the interaction of structural parameters becomes difficult, it is easier to show the combined effect of all structural parameters. The combined, pure effect is shown in Table 12. The row labeled "Interactions" is the combined sensitivity of CFD and structural uncertain parameters.

Table 12: Near field sensitivities for Green Bay atmosphere deterministic propagation.

\begin{tabular}{cccc}
\hline \hline Uncertain Parameters & ASEL & CSEL & PLdB \\
\hline Structures & $26.3 \%$ & $4.5 \%$ & $23.3 \%$ \\
CFD & $54.8 \%$ & $95.5 \%$ & $67.7 \%$ \\
Interactions & $18.9 \%$ & $\approx 0 \%$ & $9.0 \%$ \\
\hline \hline
\end{tabular}


The sensitivity analysis from the global PCE models for the deterministic standard atmosphere profile is given in Table 13. As was the case for the Green Bay atmospheric profile, angle of attack dominates the overall variance for CSEL. However, for ASEL and PLdB, the structural uncertain parameter sensitivities change significantly. Again for simplicity, the structural uncertain parameters are presented as a combined effect in Table 14.

Table 13: Expanded near field sensitivities for standard atmosphere deterministic propagation.

\begin{tabular}{cccc}
\hline \hline Uncertain Parameters & ASEL & CSEL & PLdB \\
\hline$S_{3}$ & $4.3 \%$ & - & $4.4 \%$ \\
$S_{4}$ & $4.1 \%$ & - & $4.1 \%$ \\
$S_{6}$ & - & - & $4.1 \%$ \\
$S_{1} S_{4}$ & $6.7 \%$ & - & $5.6 \%$ \\
$S_{1}$ Mach & $5.6 \%$ & - & $8.3 \%$ \\
$S_{1} \alpha$ & - & - & $2.7 \%$ \\
$S_{2} S_{3}$ & $4.5 \%$ & - & $3.2 \%$ \\
$S_{2} S_{4}$ & $4.5 \%$ & - & $3.0 \%$ \\
$S_{3} S_{5}$ & $2.8 \%$ & - & $2.8 \%$ \\
$S_{4}$ Mach & $6.0 \%$ & - & $4.6 \%$ \\
$S_{5} \alpha$ & $4.9 \%$ & - & - \\
$S_{6}$ Mach & $7.5 \%$ & - & $5.1 \%$ \\
$\alpha$ & $28.6 \%$ & $95.9 \%$ & $31.5 \%$ \\
All Others & $20.5 \%$ & $4.1 \%$ & $20.6 \%$ \\
\hline \hline
\end{tabular}

Table 14: Near field sensitivities for standard atmosphere deterministic propagation.

\begin{tabular}{cccc}
\hline \hline Uncertain Parameters & ASEL & CSEL & PLdB \\
\hline Structures & $38.7 \%$ & $1.7 \%$ & $39.8 \%$ \\
CFD & $29.5 \%$ & $97.7 \%$ & $32.3 \%$ \\
Interactions & $31.8 \%$ & $0.6 \%$ & $27.9 \%$ \\
\hline \hline
\end{tabular}

For the standard atmosphere, ASEL and PLdB responses saw an increases in sensitivity to uncertain structural parameters of $12.4 \%$ and $16.5 \%$, respectively, in comparison to the Green Bay atmosphere. This is similar to the trend observed in the previous section when comparing the two atmospheres.

\section{Local PCE Models}

To assemble the second order PCE surrogate models for the near field portion of the analysis, 90 runs were necessary. The near field waveforms from all 90 CFD runs are shown in Figure 17. The $95 \%$ confidence interval on the near field waveform from the local polynomial chaos models is given in Figure 18.

The discretization of uncertainty from the local PCE Sobol indices is shown in Figure 19 with the near field pressure waveform plotted with the same X-axis. All uncertain factors are plotted in Figure 19(b) while the combined structure uncertain factors are plotted in Figure 19(c). Throughout the waveform, individual structural uncertainties are difficult to differentiate from each other as their individual magnitudes are relatively small. As in the previous section, the impact of the structural uncertain factors is easier to understand when they are presented as a combined effect. Prior to the nose shock, the decomposition of uncertainty does not yield much useful information, as the magnitude of the overall uncertainty band is relatively small. The uncertainty in the nose shock (approximately $\mathrm{R}=375 \mathrm{in}$ ) is attributable primarily to Mach number. This is expected as the location of the nose shock peak in the near field is a function of Mach angle. The combined structures effect accounts for more variance in the first half $(\mathrm{R}<900$ in) near field 


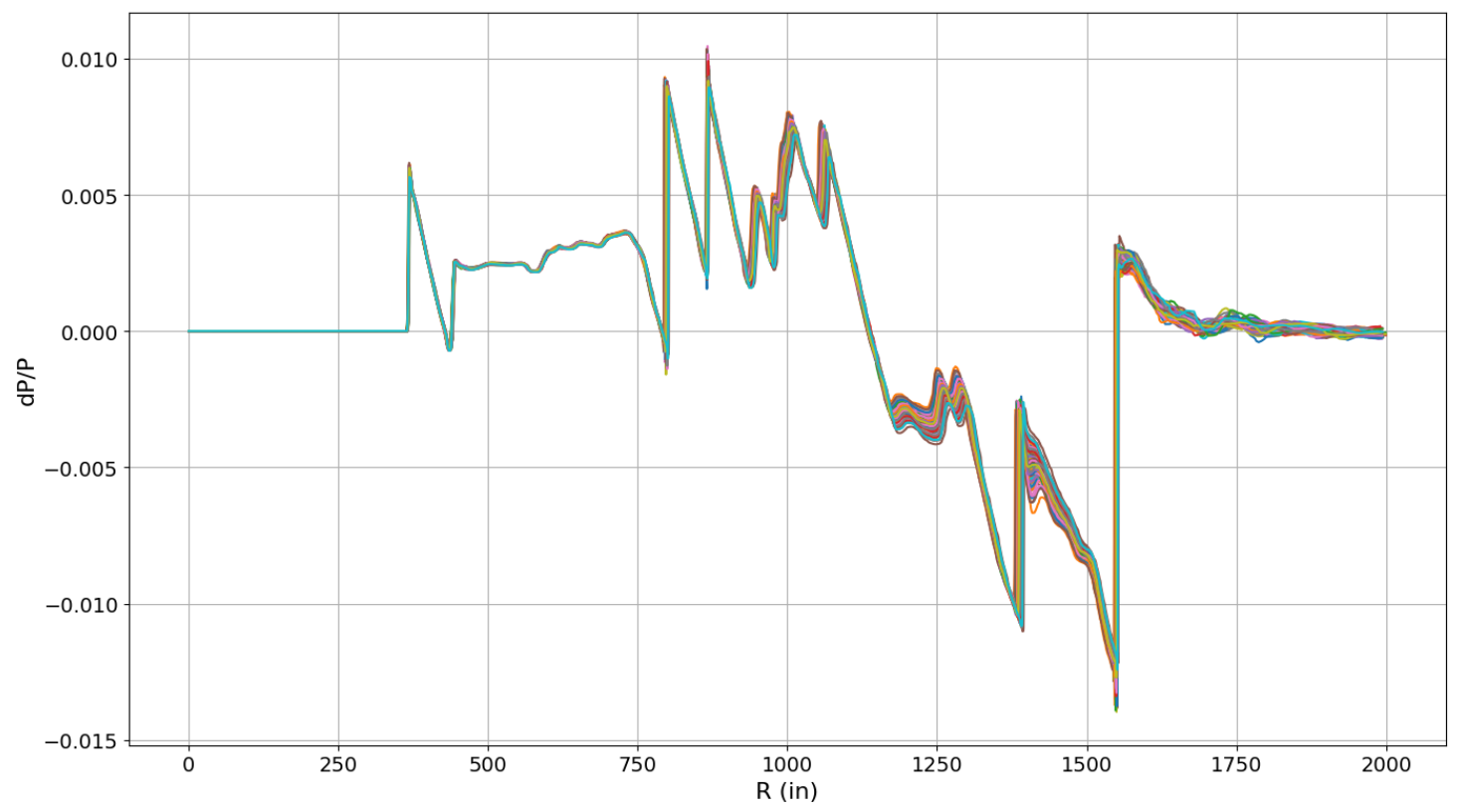

Figure 17: Near field waveforms from all runs.

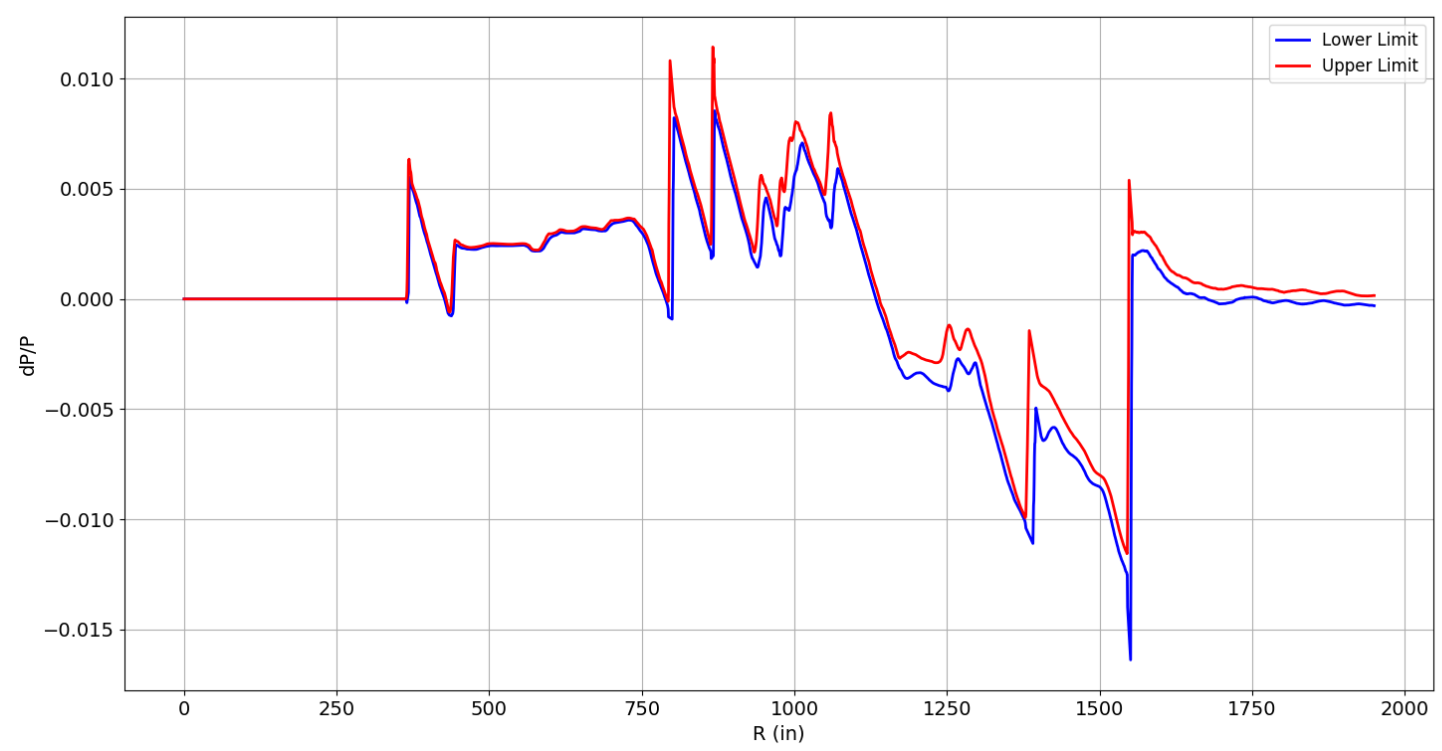

Figure 18: Near field uncertainty from local PCE models.

waveform and peaks again around $\mathrm{R}=1300$ in, which coincides with the shock structure traceable back to the wing tip. Uncertainty in angle of attack accounts for large percentages of the overall variance in the near field waveform from approximately $R=900$ in to $R=1600 \mathrm{in}$. Although this type of plot is more qualitative than quantitative, it can give another level of insight into the overall variance of the near field waveform.

\section{E. Atmospheric Propagation}

After quantifying the uncertainty in the near-field, the uncertainties in the atmospheric propagation can be incorporated into the UQ analysis for both atmospheres of interest. These results were generated from 


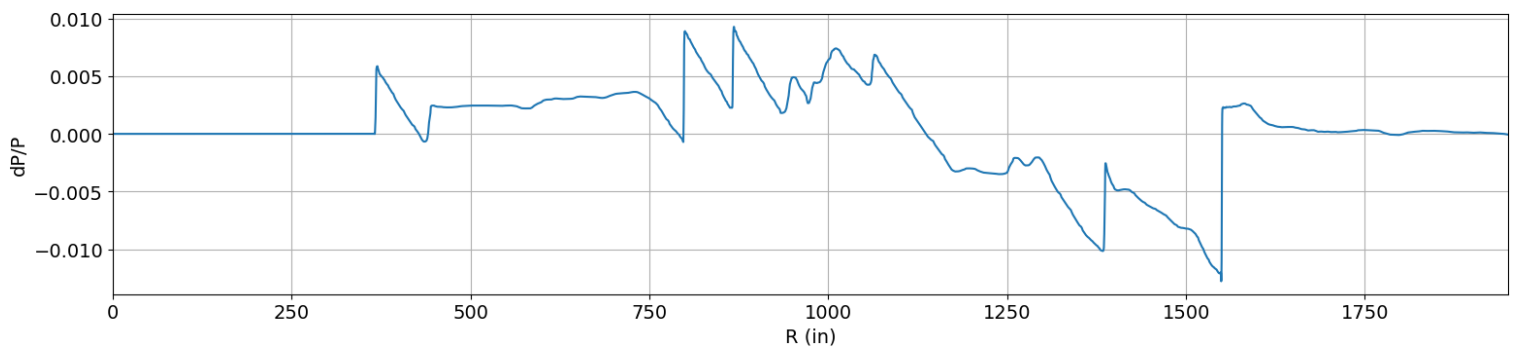

(a) Near Field Waveform

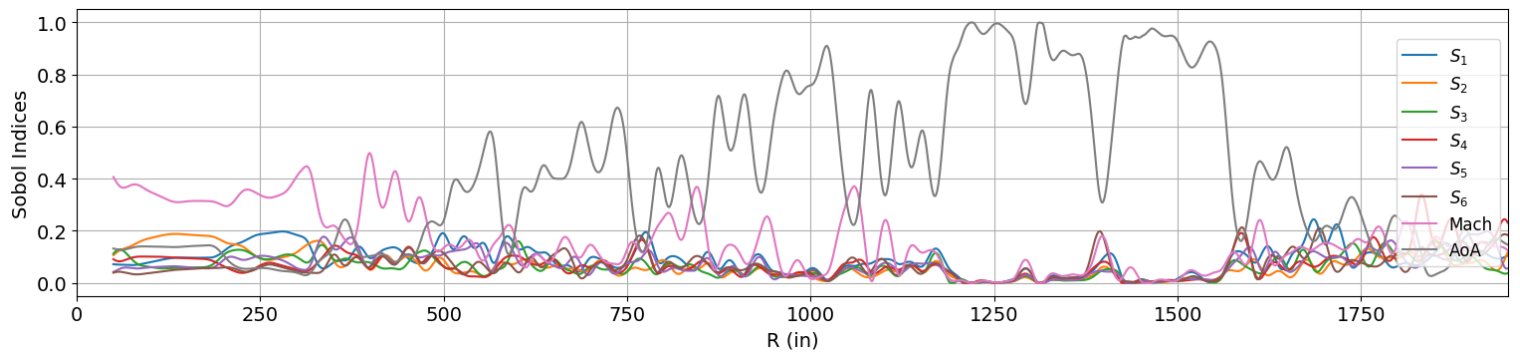

(b) Local Sobol indices

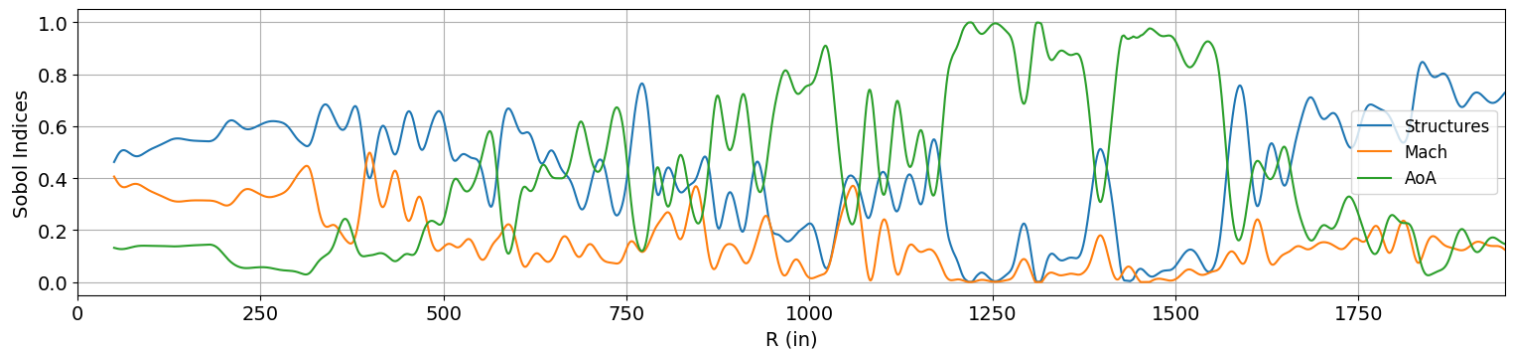

(c) Locol Sobol indices with combined structures effect

Figure 19: Discretized Sobol indices on near field waveform from local PCE models .

the Monte Carlo variance decomposition technique discussed in section II. Recall that the atmospheric uncertainties are given in Tables 2 and 3.

The probability box plots for the three ground noise metrics for the combination of the standard atmosphere is shown in Figure 20. The Sobol indices for the combination of the standard atmosphere and ground altitude interval are given in Table 15. Notice the significant contributions from the near field uncertainty, which consists of combined effects of uncertainty in the structure, Mach number, and angle of attack. Additionally, a significant contribution of the total uncertainty comes from the epistemic uncertainty sources, ground altitude and reflection factor.

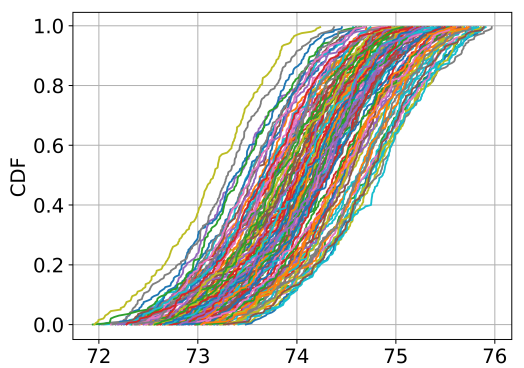

(a) ASEL.

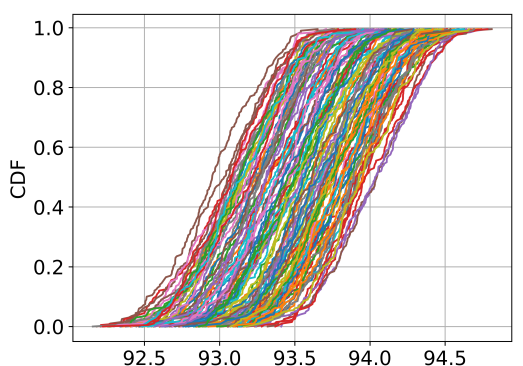

(b) CSEL.

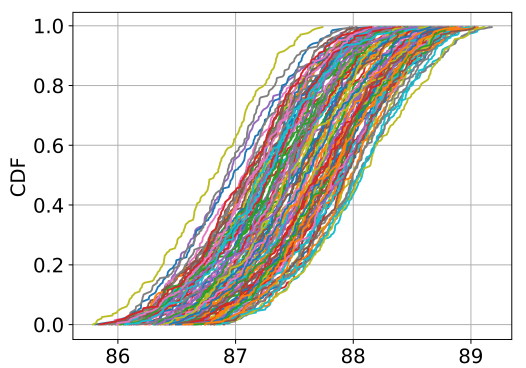

(c) PLdB.

Figure 20: Probability box for the standard atmosphere, ASEL (a), CSEL (b), PLdB (c). 
Table 15: Global variance percentages for the standard atmosphere.

\begin{tabular}{cccccc}
\hline \hline Metric & Ground Altitude & RF & RH & Temperature & Near Field \\
\hline ASEL & $18.3 \%$ & $12.4 \%$ & $\approx 0 \%$ & $\approx 0 \%$ & $69.3 \%$ \\
CSEL & $5.5 \%$ & $39.3 \%$ & $5.0 \%$ & $6.5 \%$ & $43.7 \%$ \\
PLdB & $6.8 \%$ & $21.5 \%$ & $\approx 0 \%$ & $0.4 \%$ & $71.3 \%$ \\
\hline \hline
\end{tabular}

The probability box plots for the three ground noise metrics for the combination of the Green Bay atmosphere and large ground altitude interval are shown in Figure 21. The Sobol indices for the combination of the Green Bay atmosphere and large ground altitude interval are given in Table 16. Notice in these results that the uncertainty in ASEL and PLdB is now dominated by ground altitude. The winds also play a significant role but were not present in the standard atmosphere. CSEL uncertainty is dominated by near field uncertainty.

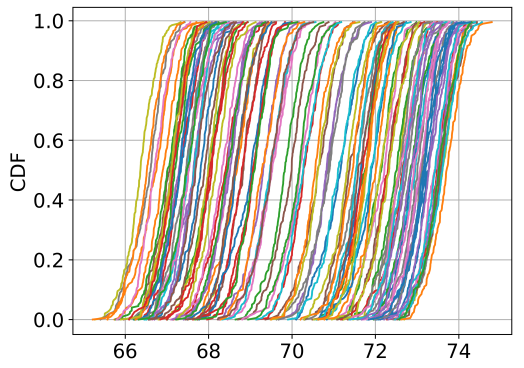

(a) ASEL.

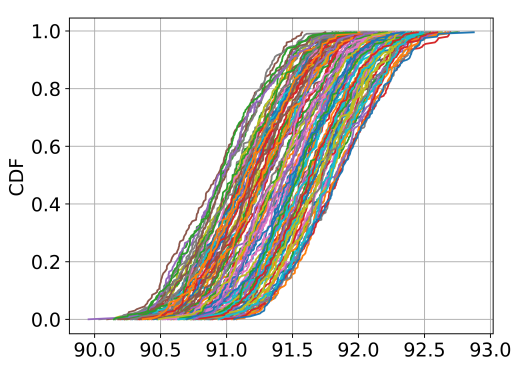

(b) CSEL.

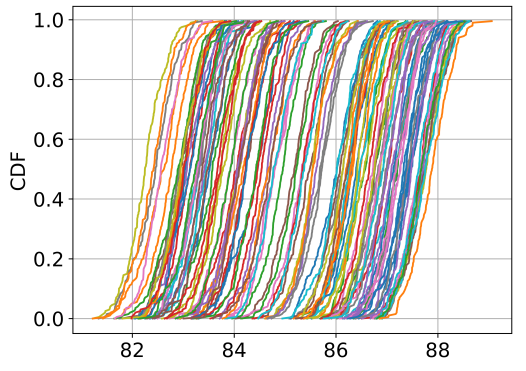

(c) PLdB.

Figure 21: Probability box for the large ground altitude interval and Green Bay atmosphere, ASEL (a), CSEL (b), PLdB (c).

Table 16: Global variance percentages for the large ground altitude interval and Green Bay atmosphere.

\begin{tabular}{cccccccc}
\hline \hline Noise Metric & Ground Altitude & RF & X-Wind & Y-wind & RH & Temperature & Near Field \\
\hline ASEL & $50.1 \%$ & $11.0 \%$ & $12.6 \%$ & $2.9 \%$ & $6.8 \%$ & $5.8 \%$ & $10.8 \%$ \\
CSEL & $\approx 0 \%$ & $34.1 \%$ & $\approx 0 \%$ & $\approx 0 \%$ & $\approx 0 \%$ & $\approx 0 \%$ & $65.9 \%$ \\
PLdB & $46.9 \%$ & $11.5 \%$ & $13.1 \%$ & $3.5 \%$ & $7.5 \%$ & $6.2 \%$ & $11.3 \%$ \\
\hline \hline
\end{tabular}

Because of the significant impact of the ground altitude, an additional analysis was performed that represents a realistic atmospheric variation of a flight near Green Bay. The probability box plots for the three ground noise metrics for the combination of the Green Bay atmosphere and small ground altitude interval are shown in Figure 22. The Sobol indices for the combination of the Green Bay atmosphere and small ground altitude interval are given in Table 17. Notice that the portion of the variance that was a result of the larger ground altitude interval is essentially transfered to the near field uncertainty contributions.

Table 17: Global variance percentages for the small ground altitude interval and Green Bay atmosphere.

\begin{tabular}{cccccccc}
\hline \hline Noise Metric & Ground Altitude & RF & X-Wind & Y-wind & RH & Temperature & Near Field \\
\hline ASEL & $26.7 \%$ & $16.9 \%$ & $13.6 \%$ & $10.7 \%$ & $5.0 \%$ & $3.6 \%$ & $23.5 \%$ \\
CSEL & $6.4 \%$ & $33.8 \%$ & $\approx 0 \%$ & $\approx 0 \%$ & $\approx 0 \%$ & $\approx 0 \%$ & $59.8 \%$ \\
PLdB & $21.9 \%$ & $19.3 \%$ & $12.4 \%$ & $9.8 \%$ & $4.4 \%$ & $3.2 \%$ & $29.0 \%$ \\
\hline \hline
\end{tabular}




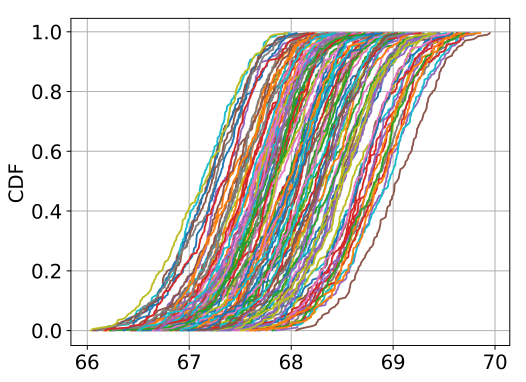

(a) ASEL.

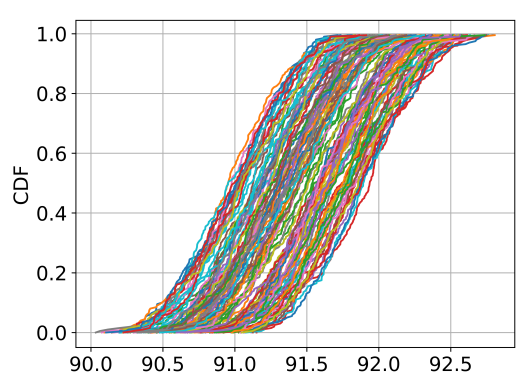

(b) CSEL.

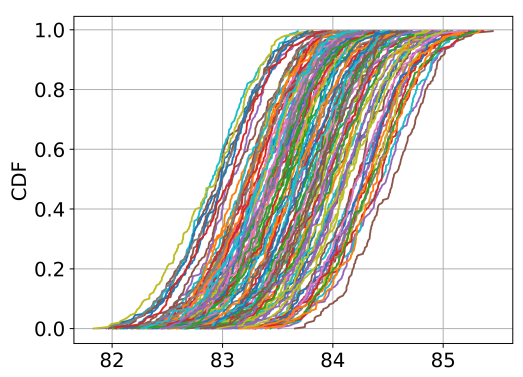

(c) PLdB

Figure 22: Probability box for the small ground altitude interval and Green Bay atmosphere, ASEL (a), CSEL (b), PLdB (c).

Evidence of non-insignificant interactions $\left(D_{T}>1\right.$ in Eq. 6) were found in the Monte Carlo variance decomposition analysis for the Green Bay atmosphere profile for both altitude interval combinations. The same evidence was not present for the standard atmosphere case. With the standard atmosphere having smaller variance percentages attributable to uncertainty in ground altitude, the interactions present in the Green Bay atmosphere cases are most likely between ground altitude and wind. The physical representation of this interaction appears to be valid, since the longer the waveform has to propagate, the longer atmospheric wind has a chance to influence the waveform. Further investigation is needed to properly characterize this possible interaction.

A summary of the $95 \%$ confidence intervals for the three atmospheric combinations is given in Table 18. The confidence interval widths for ASEL, CSEL and PLdB for the Green Bay atmosphere and large ground interval were 8.89, 2.35 and 7.15, respectively, which was approximately the same confidence interval widths found in previous research investigating the impact of uncertainty in control surface deflections on ground noise $^{8}$ with the same atmosphere profile. Although there was a shift in the central tendency for each combination, there is only a statistically significant difference for the Green Bay small altitude interval and the standard atmosphere large altitude interval for PLdB and ASEL. For the difference between cases to be statistically significant (at $a=0.05$ ), the confidence intervals must not overlap. When investigating a smaller ground altitude interval representative of a flight path, the confidence interval on PLdB decreased from 7.15 to 3.11. Other than reinforcing the finding of large sensitivities to ground altitude for PLdB, this also illustrates the progression of UQ studies. Upon the completion of an initial UQ study, resources can be directed to gain more information about uncertain factors that drive overall variation in a response. As more information becomes available about epistemic uncertain factors and their expected ranges and/or distributions, UQ studies become more valuable to decision makers.

Table 18: 95\% Confidence interval for ground noise.

\begin{tabular}{ccccc}
\hline \hline Atmosphere & Ground Interval & ASEL $(\mathrm{dB})$ & CSEL $(\mathrm{dB})$ & PLdB $(\mathrm{dB})$ \\
\hline Green Bay & {$[0,5000 \mathrm{ft}]$} & {$[65.58,74.47]$} & {$[90.26,92.61]$} & {$[81.47,88.62]$} \\
Green Bay & {$[600,1400 \mathrm{ft}]$} & {$[66.29,69.79]$} & {$[90.30,92.67]$} & {$[82.08,85.19]$} \\
Standard & {$[0,5000 \mathrm{ft}]$} & {$[72.06,75.89]$} & {$[92.35,94.58]$} & {$[85.87,89.01]$} \\
\hline \hline
\end{tabular}

\section{Conclusions}

A generalized set of procedures for determining the overall uncertainty for structural and aeroelastic uncertainties for commercial supersonic transports has been developed and applied in a case study of a low-boom concept vehicle. A robust, iterative fluid-structure interaction procedure to accurately model the uncertainties in loading conditions was developed. A screening experiment to identify the significant structural parameters was performed. Comparisons of undeformed and deformed configurations were completed. 
Two different atmospheric profiles and two ground altitude intervals were investigated. A global sensitivity analysis was performed to identify the overall drivers of uncertainty in ground noise metrics

The structural screening study performed was able to collapse the design space from 55 uncertain structural parameters to six, greatly reducing the computational resources necessary to complete this research. For ease of implementation and computational savings, this work applied uncertain structural parameters to all components in the FEM that shared the same material properties. Although the overall sensitivity to uncertain structural parameters was accurately modeled, individual structural parameters and their interactions were difficult to represent physically. In future work, assigning uncertain structural parameters by material and by component could give another level of insight into the specific uncertain structural parameters. In addition, this research has demonstrated the importance of high fidelity structural models as simplified load transferring components in the model were identified to be significant in overall displacements and ground noise. Future structural models utilized for this type of research should model load paths as accurately as possible.

Given the small displacements from the static aeroelastic deformation, the a change in ground noise of approximately $1 \mathrm{~dB}$ was not unexpected. The particular concept vehicle utilized in the case study is much more rigid than previous full scale concept vehicles researched. However, with only approximately 1 in of wing tip displacement under static aeroelastic loading, a significant ground noise difference was observed. As larger, more flexible potential low-boom vehicles enter service, the analysis performed for this research will be essential in evaluating the uncertainty in predicted ground noise generated by those vehicles.

Uncertainty in the atmospheric winds was found to be more important in the overall variation in ground noise than was found in previous research. This could be attributable, in part, to the shock structure in the aft portion of the near field pressure waveform. The strong shock emanating from the horizontal tail of an untrimmed configuration could be more influenced by atmospheric winds during propagation than the shock structure of the trimmed configuration, which does not exhibit the same strong shocks in the aft portion of the waveform. Future research should be dedicated to investigating other variance based global sensitivity methods to incorporate into this analysis to aid in characterizing interactions and higher order effects.

In summary, this research will be a crucial portion of the assessment of aeroelastic impacts of low-boom vehicles on ground noise. This work will serve as one of many building blocks for the overall system level prediction of low-boom vehicle ground noise, under uncertainty. If the prohibition on overland supersonic flight is to be repealed, it will be presumably replaced with a maximum acceptable ground noise level. To certify if a particular vehicle operating in a given atmosphere will meet this future criteria, uncertainty quantification studies like those performed in this research will play a pivotal role.

\section{Acknowledgments}

The authors would like to thank Hilmi Kamhawi for his support with the development of the automated fluid-structure interaction framework and finite element analysis.

\section{References}

\footnotetext{
${ }^{1}$ Stevens, S. S., "Perceived Level of Noise by Mark VII and Decibels (E)," The Journal of the Acoustical Society of America, Vol. 51, No. 2B, 1972, pp. 575-601.

${ }^{2}$ Rallabhandi, S. K., "Advanced Sonic Boom Prediction Using the Augmented Burgers Equation," Journal of Aircraft, Vol. 48, No. 4, July-Aug. 2011, pp. 1245-1253.

${ }^{3}$ Aftosmis, M. J., Nemec, M., and Cliff, S. E., "Adjoint-Based Low-Boom Design with Cart3D," 29th AIAA Applied Aerodynamics Conference, AIAA 2011-3500, Honolulu, Hawaii, June 2011.

${ }^{4}$ Walker, E. L., Hemsch, M. J., and West IV, T. K., "Integrated Uncertainty Quantification for Risk and Resource Management: Building Confidence in Design (Invited)," 53rd AIAA Aerospace Science Meeting, AIAA 2015-0501, Kissimmee, Florida, Jan. 2015.

${ }^{5}$ West IV, T. K., Reuter, B. W., Walker, E. L., Kleb, B., and Park, M. A., "Uncertainty Quantification and Certification Prediction of Low-Boom Supersonic Aircraft Configurations (Invited)," 32nd AIAA Applied Aerodynamics Conference, AIAA 2014-2139, Atlanta, Georgia, June 2014.

${ }^{6}$ West IV, T. K., Bretl, K. N., Walker, E. L., and Pinier, J. T., "Sonic Boom Pressure Signature Uncertainty Calculation and Propagation to Ground Noise (Invited)," 53rd AIAA Aerospace Sciences Meeting, AIAA 2015-1251, Kissimmee, Florida, Jan. 2015.

${ }^{7}$ Pinier, J. T., "Asymmetric Uncertainty Expression for High Gradient Aerodynamics," 50th AIAA Aerosciences Meeting, AIAA 2012-0081, Nashville, Tennessee, Jan. 2012.
} 
${ }^{8}$ Phillips, B. D. and West IV, T. K., "Trim Flight Conditions for a Low-Boom Aircraft Under Uncertainty," 35th AIAA Applied Aerodynamics Conference, AIAA 2017-3047, Denver, Colorado, June 2017.

${ }^{9}$ Nikbay, M., Stanford, B., West IV, T. K., and Rallabhandi, S. K., "Impact of Aeroelastic Uncertainties on the Sonic Boom Signature of a Commercial Supersonic Transport Configuration," 55th AIAA Aerospace Sciences Meeting, AIAA 2017-0040, Grapevine, Texas, Jan. 2017.

${ }^{10}$ Oberkampf, Willam L. Roy, C. J., Verification and Validation in Scientific Computing, Cambridge University Press, 2010.

${ }^{11}$ Eldred, M. and Swiler, L., "Efficient Algorithms for Mixed Aleatory-Epistemic Uncertainty Quantification with Application to Radiation-Hardened Electronics; Part I: Algorithms and Benchmark Results," Tech. Rep. SAND2009-5805, Sandia National Laboratories, September 2009.

${ }^{12}$ Hosder, S., Walters, R. W., and Balch, M., "Point-Collocation Nonintrusive Polynomial Chaos Method for Stochastic Computational Fluid Dynamics," AIAA Journal, Vol. 48, No. 12, 2010, pp. 2721-2730.

${ }^{13}$ Xiu, D. and Karniadakis, G. E., "The Wiener-Askey Polynomial Chaos for Stochastic Differential Equations," SIAM Journal on Scientific Computing, Vol. 24, No. 2, 2002, pp. 619-644.

${ }^{14}$ Ghanem, R. G. and Spanos, P. D., Stochastic Finite Elements: A Spectral Approach, Springer-Verlag, New York, 1991.

${ }^{15}$ Eldred, M. S., "Recent Advances in Non-Intrusive Polynomial Chaos and Stochastic Collocation Methods for Uncertainty Analysis and Design," AIAA Paper 2009-2274, May 2009.

${ }^{16}$ Sudret, B., "Global sensitivity analysis using polynomial chaos expansion," Reliability Engineering and System Safety, Vol. 93, No. 7, 2008, pp. 964-979.

${ }^{17}$ Saltelli, A., Annoni, P., Azzini, I., Campolongo, F., Ratto, M., and Tarantola, S., "Variance Based Sensitivity Analysis of Model Output. Design and Estimator for the Total Sensitivity Index," Computer Physics Communications, Vol. 181, 2009, pp. 259-270.

${ }^{18}$ Saltelli, A. and Boladbo, R., "An Alternative Way To Compute Fourier Amplitude Sensitivity Test (FAST)," Computational Statistics and Data Analysis, Vol. 26, No. 4, Feb. 1998, pp. 445-460.

${ }^{19}$ Sobol, I. M., "Theorems and Examples on High Dimensional Model Representation," Reliability Engineering 85 System Safety, Vol. 79, No. 2, Feb. 2003, pp. 187-193.

${ }^{20}$ Sobol, I. M., "Sensitivity Estimates for Nonlinear Mathematical Models," Mathematical Modeling and Computation Experiment, Vol. 1, 1993, pp. 407-414.

${ }^{21}$ Sobol, I., "Global Sensitivity Indices for Nonlinear Mathematical Models and their Monte Carlo Estimates," Mathematics and Computers in Simulations, Vol. 55, pp. 271-280.

${ }^{22}$ Park, M. A., Aftosmis, M. J., Campbell, R. L., Carter, M. B., Cliff, S. E., and Bangert, L. S., "Summary of the 2008 NASA Fundamental Aeronautics Program Sonic Boom Prediction Workshop," 51st AIAA Aerospace Sciences Meeting, AIAA 2013-0649, Grapevine, Texas, Jan. 2013.

${ }^{23}$ Aftosmis, M. J., Nemec, M., and Wintzer, M., "Sonic-Boom Prediction with Output-Based Adaptation and Cart3D," Fundamental Aeronautics Program Annual Meeting Sonic Boom Prediction Workshop, October 2008.

${ }^{24}$ West IV, T. K. and Gumbert, C., "Multifidelity, Multidisciplinary Design Under Uncertainty with Non-Intrusive Polynomial Chaos," 58th AIAA/ASCE/AHS/ASC Structures, Structural Dynamics, and Materials Conference, AIAA 2017-1936, Grapevine, Texas, Jan. 2017.

25 "http://www.mscsoftware.com/product/msc-nastran, "MSC Software"," May 2017.

${ }^{26}$ Samareh, J. A., "Discrete Data Transfer Technique for FluidStructure Interaction," 18th AIAA Computational Fluid Dynamics Conference, AIAA 2007-4309, Miami, Florida, June 2007.

${ }^{27}$ Shepherd, K. P. and Sullivan, B. M., "A Loudness Calculation Procedure Applied to Shaped Sonic Booms," Tech. Rep. 3134, NASA, November 1991.

${ }^{28}$ Schwartz, B. and Benjamin, S. G., "A Comparison of Temperature and Wind Measurements from ACARS-Equippped Aircraft and Rawinsondes," Weather and Forecasting, Vol. 10, Sept. 1995, pp. 528-544.

29 "Manual of the ICAO Standard Atmosphre 3rd Addition," 1993.

30 "ISO 9613-1:1993 Attenuation of Sound During Propagation Outdoors," 1993.

31 "https://lbpw.larc.nasa.gov/," Retrieved April 19, 2017. 